TRANSACTIONS OF THE

AMERICAN MATHEMATICAL SOCIETY

Volume 364, Number 9, September 2012, Pages 4605-4627

S 0002-9947(2012)05436-5

Article electronically published on April 25, 2012

\title{
CURVATURE ESTIMATES IN DIMENSIONS 2 AND 3 FOR THE LEVEL SETS OF $p$-HARMONIC FUNCTIONS IN CONVEX RINGS
}

\author{
JÜRGEN JOST, XI-NAN MA, AND QIANZHONG OU
}

\begin{abstract}
Sharp curvature estimates are given for the level sets of a class of $p$-harmonic functions in two and three dimensional convex rings.
\end{abstract}

\section{INTRODUCTION}

The geometry of the solutions of a partial differential equation is an important issue. The convexity of the level sets of the solutions of elliptic partial differential equations has been studied for a long time. For instance, Ahlfors [1 contains the well-known result that the level curves of the Green function on a simply connected convex domain in the plane are the convex Jordan curves. In 1956, Shiffman 20] proved several beautiful theorems concerning the geometry of a minimal annulus whose boundary consists of two closed convex curves in parallel planes $P_{1}, P_{2}$. One of his theorems stated that the intersection of the surface with any plane $P$, between $P_{1}$ and $P_{2}$, is a convex Jordan curve; in particular, it follows that this surface is embedded. For elliptic partial differential equations on domains in $\mathbb{R}^{n}$, the problem of level set convexity was first considered by Gabriel. In 1957, Gabriel [9] proved that the level sets of the Green function on a three dimensional convex domain are strictly convex. Later, in 1977, Lewis 14 extended Gabriel's result to p-harmonic functions in higher dimensions and obtained the following theorem.

Theorem 1.1 (Gabriel [9] and Lewis [14]). Let u satisfy

$$
\begin{cases}\operatorname{div}\left(|D u|^{p-2} D u\right)=0 & \text { in } \Omega=\Omega_{0} \backslash \bar{\Omega}_{1}, \\ u=0 & \text { on } \partial \Omega_{0}, \\ u=1 & \text { on } \partial \Omega_{1},\end{cases}
$$

where $1<p<+\infty, \Omega_{0}$ and $\Omega_{1}$ are smooth bounded convex domains in $\mathbb{R}^{n}, n \geq 2$, $\bar{\Omega}_{1} \subset \Omega_{0}$. (We say that u satisfies the homogeneous Dirichlet boundary conditions in the convex ring $\Omega=\Omega_{0} \backslash \bar{\Omega}_{1}$.) Then all the level sets of $u$ are strictly convex in $\Omega$.

In 1982, Caffarelli-Spruck [5] generalized the Lewis [14 results to a class of semilinear elliptic partial differential equations. A survey of this subject is given by Kawohl [13]. For more recent extensions, see Greco [12] and Cuoghi-Salani [6].

Received by the editors January 12, 2009 and, in revised form, July 20, 2010.

2010 Mathematics Subject Classification. Primary 35J05.

Key words and phrases. Curvature estimates, level sets, harmonic function.

The research of the second author was supported by NSFC No.10671186 and "BaiRen Program in Chinese Academy of Sciences".

The research of the third author was supported by Guangxi Natural Science Foundation 2010GXNSFA013123. 
The aforementioned results are of a qualitative nature. This naturally leads us to the question of quantitative results, that is, estimates for the curvature of the level sets of the solutions of such elliptic problems. This is the topic of the present paper.

For a two dimensional harmonic function defined on a convex ring with homogeneous Dirichlet boundary conditions, by the theorem of Lewis [14, as explained, the level set of this function is strictly convex. Ortel-Schneider [18] and Longinetti [16] proved that the curvature of the level curves attains its minimum on the boundary (see Talenti 21] for related results). Later, in 1987, Longinetti [17] used the same technique to obtain a similar theorem for minimal surfaces, where the convexity of the level sets follows from the theorem of Shiffman [20].

On the other hand, in 1989, Rosay-Rudin [19] proposed a new measure for the convexity of the level sets, and showed that with regard to this measure, the level sets of the harmonic functions on a convex ring in any dimension with homogeneous Dirichlet boundary conditions are "more convex" in the interior than on the boundaries.

In this paper, we study the level sets of $p$-harmonic functions in two and three dimensions, and we obtain quantitative estimates for the convexity of the level sets. Our main results are as follows. We first state the two dimensional case.

Theorem 1.2. Let $\Omega$ be a smooth bounded domain in $\mathbb{R}^{2}$, and $u$ be a smooth p-harmonic function in $\Omega$, i.e.

$$
\operatorname{div}\left(|D u|^{p-2} D u\right)=0 \quad \text { in } \Omega .
$$

Assume $|D u| \neq 0$ in $\Omega$. If $\frac{3}{2} \leq p \leq 3$ and the level lines of $u$ are convex with respect to the normal $D u$, then the curvature of the level lines of $u$ attains its minimum on $\partial \Omega$.

In three dimensions, we have the following result.

Theorem 1.3. Let $\Omega$ be a smooth bounded domain in $\mathbb{R}^{3}$ and $u$ be a smooth $p$ harmonic function in $\Omega$, which satisfies (1.2). Assume $|D u| \neq 0$ in $\Omega$. If $2 \leq p<$ $+\infty$ and the level sets of $u$ are all strictly convex with respect to the normal Du, then the Gaussian curvature of the level sets of $u$ attains its minimum on $\partial \Omega$.

We can apply Theorem 1.2 and Theorem 1.3 to obtain the following version of Lewis's theorem [14].

Corollary 1.4. Let $u$ be the solution of the following boundary value problem,

$$
\begin{cases}\operatorname{div}\left(|D u|^{p-2} D u\right)=0 & \text { in } \Omega=\Omega_{0} \backslash \bar{\Omega}_{1}, \\ u=0 & \text { on } \partial \Omega_{0}, \\ u=1 & \text { on } \partial \Omega_{1},\end{cases}
$$

where $\Omega$ is a smooth convex ring as in Theorem 1.1. If $n=2$ and $\frac{3}{2} \leq p \leq 3$, then the curvature of the level lines of $u$ attains its minimum on $\partial \Omega$. If $n=3$ and $2 \leq p<+\infty$, then the Gaussian curvature of the level sets of $u$ attains its minimum on $\partial \Omega$.

Rosay-Rudin [19] apply the maximum principle directly to quantities formed by the solution $u$ to obtain their results. This works because convexity of a function can be expressed in terms of this function itself without involving derivatives. Our method is different and depends on new curvature inequalities. This is in line 
with general strategies for understanding solutions of elliptic partial differential equations from a deeper geometric perspective. These inequalities represent the main achievement of the present paper. More precisely, we show that the Gaussian curvature $K$ of the level sets satisfies the following $p$-Laplacian inequality:

$$
a^{i j}(D u) K_{i j}+b^{i} K_{i} \leq 0 \quad \text { in } \Omega,
$$

where

$$
a^{i j}(D u):=|D u|^{2} \delta_{i j}+(p-2) u_{i} u_{j}
$$

with $b^{i}$ being locally bounded. Then we can apply the strong maximum principle [10] to obtain our results.

The results obtained here are for dimensions 2 and 3 . In this regard, we note that sometimes lower dimensional harmonic functions display certain phenomena that are not present in the higher dimensional case. For example in three dimensions, the famous theorem of Lewy [15] states that if $u$ is a harmonic function on a domain in $\mathbb{R}^{3}$ and the map $x \longrightarrow D u(x)$ is a homeomorphism, then $x \longrightarrow D u(x)$ is a diffeomorphism. In 1991, Gleason-Wolff [11] extended this results to higher dimensions, but needed some extra conditions, and gave a counterexample in the higher dimensional case without these additional conditions.

Nevertheless, it seems that, in principle, our method is of a general nature and that, therefore, some extension of it should also give some results in higher dimensional cases.

From the standard theory of elliptic partial differential equations, we know that the (unique) solution of equation (1.2) is smooth in any domain $\Omega$ in $\mathbb{R}^{n}, n \geq 2$ when $|D u| \neq 0$ in $\Omega$. This is verified in detail by Lewis [14].

In section 2, we first give a detailed definition of the convexity of the level sets which appeared in [2, then obtain the curvature formulas for the Gaussian curvature of the level sets of a function. The proofs of our theorems, as given in the sequel, depend on difficult calculations. The main technique consists in rearranging third derivative terms using the equation and the first derivatives condition for the Gaussian curvature. In dimensions 2 and 3, we get "good" signs for the second and third derivative terms, which allows us to reach our conclusions.

\section{The CURVATURE FORMUlas OF LEVEL SETS}

In this section, we first recall some fundamental notation in classical surfaces theory and provide the definitions for the convexity of surfaces in Euclidean space with respect to the upward normal. Then we introduce the level sets of a function $u$, and we derive the curvature formulas of the level sets of $u$, which appeared in [2]. For convenience, we will adapt the following convention for indices in this section:

$$
1 \leq i, j, \ldots \leq n-1 ; \quad 1 \leq \alpha, \beta, \ldots \leq n,
$$

and the repeated indices are summed unless stated otherwise.

2.1. Classical differential geometry of graph and its convexity. First we recall some fundamental notation in classical surface theory as in [7. Assume a surface $\Sigma \subset \mathbb{R}^{n}$ is given by the graph of a $C^{2}$ function $v$ in a domain in $\mathbb{R}^{n-1}$ :

$$
x_{n}=v\left(x^{\prime}\right), x^{\prime}=\left(x_{1}, x_{2}, \ldots, x_{n-1}\right) \in \mathbb{R}^{n-1} .
$$


The first fundamental form for the graph of $x_{n}=v\left(x^{\prime}\right)$ is given by

$$
g_{i j}=\delta_{i j}+v_{i} v_{j} \text {. }
$$

The upward normal direction $\vec{n}$ and the second fundamental form II w.r.t. $\vec{n}$ for the graph $x_{n}=v\left(x^{\prime}\right)$ are respectively given by

$$
\begin{aligned}
\vec{n} & =\frac{1}{\sqrt{1+|D v|^{2}}}\left(-v_{1},-v_{2}, \ldots,-v_{n-1}, 1\right), \\
\mathbb{I} & =\left(b_{i j}\right)=\left(\frac{v_{i j}}{W}\right),
\end{aligned}
$$

where $1 \leq i, j, k, l \leq n-1$ and $W=\left(1+|D v|^{2}\right)^{\frac{1}{2}}$.

Now we recall the definition of a convex surface in classical differential geometry 7].

Definition 2.1. The graph of a function $x_{n}=v\left(x^{\prime}\right)$ is convex with respect to the upward normal $\vec{n}$ as in (2.3) if the second fundamental form $\mathbb{I}:=\left(b_{i j}\right)=\left(\frac{v_{i j}}{W}\right)$ of the graph $x_{n}=v\left(x^{\prime}\right)$ is nonnegative definite.

The principal curvatures $\kappa=\left(\kappa_{1}, \kappa_{2}, \ldots, \kappa_{n-1}\right)$ of the graph of $v$, being the eigenvalues of the second fundamental form relative to the first fundamental form, satisfy

$$
\operatorname{det}\left(b_{i j}-\kappa_{k} g_{i j}\right)=0,
$$

or equivalently,

$$
\operatorname{det}\left(a_{i j}-\kappa_{k} \delta_{i j}\right)=0
$$

with

$$
\left(a_{i j}\right)=\left(g^{i j}\right)^{\frac{1}{2}}\left(b_{i j}\right)\left(g^{i j}\right)^{\frac{1}{2}}
$$

where $\left(g^{i j}\right)$ is the inverse matrix to $\left(g_{i j}\right)$ and $\left(g^{i j}\right)^{\frac{1}{2}}$ is its positive square root, and there is no sum for $i, j$. They are given explicitly by

$$
\begin{aligned}
\left(g^{i j}\right) & =\left(\delta_{i j}-\frac{v_{i} v_{j}}{W^{2}}\right), \\
\left(g^{i j}\right)^{\frac{1}{2}} & =\left(\delta_{i j}-\frac{v_{i} v_{j}}{W(1+W)}\right) .
\end{aligned}
$$

Then we have the following well-known formulas.

Lemma 2.2 (4]). The principal curvatures of the graph $x_{n}=v\left(x^{\prime}\right)$ with respect to the upward unit normal (2.3) are the eigenvalues of the symmetric curvature matrix

$$
\left(a_{i l}\right)=\frac{1}{W}\left(v_{i l}-\frac{v_{i} v_{j} v_{j l}}{W(1+W)}-\frac{v_{l} v_{k} v_{k i}}{W(1+W)}+\frac{v_{i} v_{l} v_{j} v_{k} v_{j k}}{W^{2}(1+W)^{2}}\right) .
$$

2.2. The convexity of the level sets of a function. Now we give the definition of the level sets of the function $u$.

For a domain $\Omega \subset \mathbb{R}^{n}$ and $u \in C^{2}(\Omega)$, we shall use the following notation.

Definition 2.3. Assuming $|D u| \neq 0$ in $\Omega$, we denote the level set of $u$ through a point $x_{o} \in \Omega$ by $\Sigma^{u\left(x_{o}\right)}:=\left\{x \in \Omega \mid u(x)=u\left(x_{o}\right)\right\}$.

Now we shall locally work near such a point $x_{o}$, where $\left|D u\left(x_{o}\right)\right| \neq 0$. We first state the definition of the convexity for the level sets $\Sigma^{u\left(x_{o}\right)}$ in this special case. Without loss of generality we assume $u_{n}\left(x_{o}\right) \neq 0$ and work on a small neighborhood of $x_{0}$. 
By the implicit function theorem, locally the level set $\Sigma^{u\left(x_{o}\right)}$ can be represented as (2.1), where the function $v\left(x^{\prime}\right)$ satisfies the following equation:

$$
u\left(x_{1}, x_{2}, \ldots, x_{n-1}, v\left(x_{1}, x_{2}, \ldots, x_{n-1}\right)\right)=u\left(x_{o}\right) .
$$

It follows that

$$
u_{i}+u_{n} v_{i}=0
$$

and hence

$$
v_{i}=-\frac{u_{i}}{u_{n}}
$$

From (2.10), we have

$$
W=\left(1+|D v|^{2}\right)^{\frac{1}{2}}=\frac{|D u|}{\left|u_{n}\right|} .
$$

Using (2.3) and (2.11), it follows that the upward normal direction of the level sets is

$$
\vec{n}=\frac{\left|u_{n}\right|}{|D u| u_{n}}\left(u_{1}, u_{2}, \ldots, u_{n-1}, u_{n}\right) .
$$

Now differentiating (2.9) again, we have

$$
u_{i j}+u_{i n} v_{j}+u_{n j} v_{i}+u_{n n} v_{i} v_{j}+u_{n} v_{i j}=0 \text {. }
$$

Then

$$
\begin{aligned}
v_{i j} & =-\frac{1}{u_{n}}\left[u_{i j}+u_{i n} v_{j}+u_{n j} v_{i}+u_{n n} v_{i} v_{j}\right] \\
& =-\frac{1}{u_{n}^{3}}\left[u_{n}^{2} u_{i j}+u_{n n} u_{i} u_{j}-u_{n} u_{j} u_{i n}-u_{n} u_{i} u_{j n}\right] .
\end{aligned}
$$

If we let

$$
h_{i j}=u_{n}^{2} u_{i j}+u_{n n} u_{i} u_{j}-u_{n} u_{j} u_{i n}-u_{n} u_{i} u_{j n}
$$

it follows that

$$
v_{i j}=-\frac{h_{i j}}{u_{n}^{3}} .
$$

From (2.11) and (2.16), with respect to the upward normal direction (2.12), the second fundamental form $\mathbb{I}$ of the level surface of the function $u$ is

$$
\left(b_{i j}\right)=\left(\frac{v_{i j}}{W}\right)=\left(-\frac{\left|u_{n}\right| h_{i j}}{|D u| u_{n}^{3}}\right) .
$$

From Definition 2.1, we give the definition of the convexity for the level sets $\Sigma^{u\left(x_{o}\right)}=\left\{x \in \Omega \mid u(x)=u\left(x_{o}\right)\right\}$ of the function $u(x)$, where $|D u|\left(x_{o}\right) \neq 0$ in $\Omega$.

Definition 2.4. For the function $u(x) \in C^{2}(\Omega)$ we assume $|D u| \neq 0$ in $\Omega$, and locally we can let $u_{n}\left(x_{o}\right) \neq 0$ for $x_{o} \in \Omega$. We define locally that the level set $\Sigma^{u\left(x_{0}\right)}=\left\{x \in \Omega \mid u(x)=u\left(x_{o}\right)\right\}$ is convex if the second fundamental form (2.17) is nonnegative definitive with respect to the upward normal direction (2.12).

Remark 2.5. At $x_{o} \in \Omega$, with $|D u|\left(x_{o}\right) \neq 0$, without loss of generality we may then assume $u_{n}\left(x_{o}\right) \neq 0$.

By choosing the coordinate system suitably, we may assume $\frac{D u}{|D u|}$ to be the upward normal of the level set $\Sigma^{u\left(x_{o}\right)}$ at $x_{o}$. Then $u_{n}\left(x_{o}\right)>0$ by (2.12), and from 
Definition 2.4, if the level set $\Sigma^{u\left(x_{o}\right)}$ at $x_{o}$ is convex with respect to the normal $\frac{D u}{|D u|}$, then the matrix $\left(h_{i j}\left(x_{o}\right)\right)$ is nonpositive definite.

On the other hand, if we choose $-\frac{D u}{|D u|}$ to be the upward normal of the level set $\Sigma^{u\left(x_{o}\right)}$ at $x_{o}$, then $u_{n}\left(x_{o}\right)<0$ by (2.12), and from Definition 2.4, if the level set $\Sigma^{u\left(x_{o}\right)}$ at $x_{o}$ is convex with respect to the normal $-\frac{D u}{|D u|}$, the matrix $h_{i j}\left(x_{o}\right)$ is nonnegative definite.

In the remainder of this paper, we always choose $\frac{D u}{|D u|}$ to be the upward normal of the level sets of $u$.

2.3. The curvature formulas of level sets of functions. Now we obtain the representation of the curvature matrix $\left(a_{i j}\right)$ of the level sets of the function $u$ by the derivatives of $u$, and deduce the curvature formulas for the level sets of $u$. We work locally near $x_{0} \in \Omega$ with $u_{n}\left(x_{0}\right)>0$.

From (2.7), (2.11) and (2.17) it follows that the entries of the symmetric curvature matrix $\left(a_{i j}\right)$ become

$$
a_{i j}=\frac{1}{|D u| u_{n}^{2}}\left\{-h_{i j}+\frac{u_{i} u_{l} h_{j l}}{W(1+W) u_{n}^{2}}+\frac{u_{j} u_{l} h_{i l}}{W(1+W) u_{n}^{2}}-\frac{u_{i} u_{j} u_{k} u_{l} h_{k l}}{W^{2}(1+W)^{2} u_{n}^{4}}\right\}
$$

We put

$$
\begin{aligned}
B_{i j}: & =\frac{u_{i} u_{l} h_{j l}}{W(1+W) u_{n}^{2}}+\frac{u_{j} u_{l} h_{i l}}{W(1+W) u_{n}^{2}}, \\
C_{i j}: & =\frac{u_{i} u_{j} u_{k} u_{l} h_{k l}}{W^{2}(1+W)^{2} u_{n}^{4}}
\end{aligned}
$$

and

$$
A_{i j}:=-h_{i j}+B_{i j}-C_{i j}
$$

Thus,

$$
a_{i j}=\frac{1}{|D u| u_{n}^{2}} A_{i j}
$$

For the $C^{2}$ hypersurface $\Sigma^{c}$ in $\mathbb{R}^{n}$, we let $\kappa=\left(\kappa_{1}, \ldots, \kappa_{n-1}\right)$ be its principal curvatures. For $m=1, \ldots, n-1$, the $m$-th curvature of $\Sigma^{c}$ is defined by

$$
\sigma_{m}\left[\Sigma^{c}\right]=\sigma_{m}\left(\kappa_{1}, \ldots, \kappa_{n-1}\right),
$$

where $\sigma_{m}$ is the $m$-th elementary symmetric function; that is, for $1 \leq m \leq n-1$ and $\lambda=\left(\lambda_{1}, \ldots, \lambda_{n-1}\right) \in \mathbb{R}^{n-1}$,

$$
\sigma_{m}(\lambda)=\sum_{1 \leq i_{1}<\ldots<i_{m} \leq n-1} \lambda_{i_{1}} \cdots \lambda_{i_{m}}
$$

For an $(n-1) \times(n-1)$ symmetric matrix $V=\left(V_{i j}\right)$, we define

$$
\sigma_{m}(V)=\sum_{1 \leq i_{1}<\ldots<i_{m} \leq n-1} \operatorname{sgn}(\sigma) V_{i_{1} i_{\sigma(1)}} \cdots V_{i_{m} i_{\sigma(m)}} .
$$

Obviously

$$
\sigma_{m}(V)=\sigma_{m}(\lambda)
$$


with $\lambda=\lambda(V)$ being the eigenvalues of $V$. By the way, let $F=F(M)$ be a $C^{2}$ function of the entries of an $m \times m$ matrix $M=\left(M_{i j}\right)$. Then the following notation may also be used in the next two sections:

$$
F^{i j}:=\frac{\partial F}{\partial M_{i j}}, \quad F^{i j, r s}:=\frac{\partial^{2} F}{\partial M_{i j} \partial M_{r s}} .
$$

The principal curvatures of the level set $\Sigma^{u\left(x_{o}\right)}$ of the function $u \in C^{2}(\Omega)$ then are the eigenvalues of the $(n-1) \times(n-1)$ symmetric matrix

$$
\left(a_{i j}\right)=\left(\frac{1}{|D u| u_{n}^{2}} A_{i j}\right)
$$

The $m$-th curvatures are given by

$$
\sigma_{m}\left[\Sigma^{c}\right]=\sigma_{m}\left(\kappa_{1}, \ldots, \kappa_{n-1}\right)=\left(\frac{1}{|D u| u_{n}^{2}}\right)^{m} \sigma_{m}\left(A_{i j}\right)
$$

where there is no sum for $1 \leq m \leq n-1$.

Proposition 2.6. Let $u(x) \in C^{2}(\Omega)$ for $\Omega \subset \mathbb{R}^{n}$ and $|D u| \neq 0$ in $\Omega$. Then the $k$-th curvature of the level set $\Sigma^{c}$, w.r.t. the normal Du, is

$$
\sigma_{k}\left[\Sigma^{c}\right]=(-1)^{k} \frac{\partial \sigma_{k+1}\left(D^{2} u\right)}{\partial u_{\alpha \beta}} u_{\alpha} u_{\beta}|D u|^{-(k+2)}
$$

where $1 \leq k \leq n-1$ and there is no sum for $k$ on the right of (2.24).

Remark 2.7. In [22], Trudinger obtained a similar formula for the $k$-th curvature of the level set $\Sigma^{c}$.

Proof. We first prove that the formula (2.24) is independent of the choice of the coordinate system $\left\{x_{\alpha}\right\}$ of $\mathbb{R}^{n}$. Then we calculate (2.24) for a special coordinate system.

Step 1. Let $P=\left(p_{\alpha \beta}\right)$ be an orthogonal transformation between two coordinate systems, i.e. $\left(\bar{x}_{1}, \cdots, \bar{x}_{n}\right)=\left(x_{1}, \cdots, x_{n}\right) P$. Define $\bar{u}(\bar{x})=u\left(\bar{x} P^{T}\right)$. Denote by $D \bar{u}=\left(\bar{u}_{1}, \cdots, \bar{u}_{n}\right)$ and $D^{2} \bar{u}=\left(\bar{u}_{\alpha \beta}\right)$ the gradient and the Hessian respectively of $\bar{u}$ w.r.t. $\left(\bar{x}_{1}, \cdots, \bar{x}_{n}\right)$. Then we have

$$
\begin{array}{r}
\left(\bar{u}_{1}, \cdots, \bar{u}_{n}\right)=\left(u_{1}, \cdots, u_{n}\right) P \\
\left(\bar{u}_{\alpha \beta}\right)=P^{T}\left(u_{\alpha \beta}\right) P=P^{-1}\left(u_{\alpha \beta}\right) P, \\
\left(u_{\alpha \beta}\right)=P\left(\bar{u}_{\alpha \beta}\right) P^{T} \Rightarrow \frac{\partial u_{\gamma \delta}}{\partial \bar{u}_{\alpha \beta}}=p_{\gamma \alpha} p_{\delta \beta} .
\end{array}
$$


Clearly $|D u|$ and $\sigma_{k}\left(D^{2} u\right)$ are the invariants of $P$; i.e., $|\bar{D} u|=|D u|$ and $\sigma_{k}\left(D^{2} \bar{u}\right)$ $=\sigma_{k}\left(D^{2} u\right)$ for $1 \leq k \leq n-1$. Therefore we can derive

$$
\begin{aligned}
\frac{\partial \sigma_{k+1}\left(D^{2} \bar{u}\right)}{\partial \bar{u}_{\alpha \beta}} \bar{u}_{\alpha} \bar{u}_{\beta} & =\frac{\partial \sigma_{k+1}\left(D^{2} u\right)}{\partial u_{\gamma \delta}} \frac{\partial u_{\gamma \delta}}{\partial \bar{u}_{\alpha \beta}}\left(u_{\lambda} p_{\lambda \alpha}\right)\left(u_{\mu} p_{\mu \beta}\right) \\
& =\frac{\partial \sigma_{k+1}\left(D^{2} u\right)}{\partial u_{\gamma \delta}} p_{\gamma \alpha} p_{\delta \beta} u_{\lambda} p_{\lambda \alpha} u_{\mu} p_{\mu \beta} \\
& =\frac{\partial \sigma_{k+1}\left(D^{2} u\right)}{\partial u_{\gamma \delta}} u_{\lambda} u_{\mu}\left(p_{\gamma \alpha} p_{\lambda \alpha}\right)\left(p_{\delta \beta} p_{\mu \beta}\right) \\
& =\frac{\partial \sigma_{k+1}\left(D^{2} u\right)}{\partial u_{\gamma \delta}} u_{\lambda} u_{\mu} \delta_{\gamma \lambda} \delta_{\delta \mu} \cdots \cdots \text { for } P \text { an orthogonal matrix } \\
& =\frac{\partial \sigma_{k+1}\left(D^{2} u\right)}{\partial u_{\gamma \delta}} u_{\gamma} u_{\delta} .
\end{aligned}
$$

The above calculations show that the right side of (2.24) is invariant under orthogonal transformations.

Step 2. For any point $x_{0} \in \Omega$, we choose a special coordinate systerm such that $|D u|=u_{n}>0$ and $\left(u_{i j}\right)$ (and hence $A_{i j}$ by (2.20) ) is diagonal. Therefore the $\sigma_{k}$ curvature of the level sets is

$$
\begin{aligned}
\sigma_{k}\left[\Sigma^{u\left(x_{0}\right)}\right] & =\sigma_{k}\left(\kappa_{1}, \ldots, \kappa_{n-1}\right)=\left(\frac{1}{|D u| u_{n}^{2}}\right)^{k} \sigma_{k}\left(A_{i j}\right) \\
& =\frac{(-1)^{k}}{|D u|^{k}} \frac{\partial \sigma_{k+1}\left(D^{2} u\right)}{\partial u_{n n}} \\
& =(-1)^{k} \frac{\partial \sigma_{k+1}\left(D^{2} u\right)}{\partial u_{n n}} u_{n} u_{n}|D u|^{-(k+2)} .
\end{aligned}
$$

Note that there is no sum for $1 \leq k \leq n-1$.

From Step 1 and Step 2, we get the proof of the curvature formula (2.24).

\section{Proof of Theorem 1.2}

Let $u$ be a solution of (1.2) and $|D u| \neq 0$ in $\Omega$. Assume the level lines of $u$ are convex with respect to the normal $D u$. Then, by (2.24), the curvature of the level lines of $u$ is

$$
K=-F^{i j} u_{i} u_{j}|D u|^{-3}
$$

where $F^{i j}$ is as in (2.22) with $F:=\operatorname{det}\left(D^{2} u\right)$. In this section, the repeated indices are summed from 1 to 2 , unless stated otherwise.

By (1.5), (1.2) is equivalent to

$$
a^{i j} u_{i j}=0 \text { in } \Omega .
$$

At any fixed point $x \in \Omega$, we will prove the following elliptic inequality:

$$
a^{i j} K_{i j}+b^{i} K_{i} \leq 0
$$

with $b^{i}$ locally bounded. Then by the strong maximum principle [10, we can get our result, Theorem [1.2, immediately.

Differentiating both sides of (3.1), we deduce:

$$
K_{\alpha}=-F^{i j, r s} u_{r s \alpha} u_{i} u_{j}|D u|^{-3}-2 F^{i j} u_{i \alpha} u_{j}|D u|^{-3}+3 F^{i j} u_{i} u_{j} u_{k} u_{k \alpha}|D u|^{-5} \text {. }
$$


Next, all the calculations will be done at the fixed point $x$. In order to prove (3.3) at an arbitrary point $x \in \Omega$, as in Caffarelli-Friedman [3], we choose the normal coordinate at $x$. By rotating the coordinate system suitably by $T_{x}$, we may assume that $u_{1}(x)=0$ and $u_{2}(x)=|\nabla u|>0$. We can further assume that $u_{11}(x)<0$. We also choose $T_{x}$ to vary smoothly with $x$. If we can establish (3.3) at $x$ under the above assumptions, then going back to the original coordinate we find that (3.3) remains valid with new locally bounded coefficients on $\nabla K$ in (3.3), depending smoothly on the independent variables. Thus it suffices to establish (3.3) under the above assumptions.

From now on, all the calculations will be done at the fixed point $x$. So we can get by (3.4):

$$
K_{\alpha}=-F^{22, r s} u_{r s \alpha} u_{2}^{2}|D u|^{-3}-2 F^{i 2} u_{i \alpha} u_{2}|D u|^{-3}+3 F^{22} u_{2}^{3} u_{2 \alpha}|D u|^{-5}
$$

and

$$
\begin{aligned}
|D u|^{3} K_{\alpha \beta}= & -F^{22, r s} u_{r s \alpha \beta} u_{2}^{2}-2 F^{i 2} u_{i \alpha \beta} u_{2}+3 F^{22} u_{2} u_{2 \alpha \beta} \\
& -4 F^{i 2, r s} u_{r s \alpha} u_{i \beta} u_{2}+3 F^{22, r s} u_{r s \alpha} u_{2} u_{2 \beta}+3 F^{22, r s} u_{r s \beta} u_{2} u_{2 \alpha} \\
& -2 F^{i j} u_{i \alpha} u_{j \beta}+6 F^{i 2} u_{i \alpha} u_{2 \beta}+6 F^{i 2} u_{i \beta} u_{2 \alpha} \\
& +3 F^{22} u_{k \alpha} u_{k \beta}-15 F^{22} u_{2 \alpha} u_{2 \beta} .
\end{aligned}
$$

Multiplying by $a^{\alpha \beta}$ on both sides of the above equality, we have

$$
\begin{aligned}
|D u|^{3} a^{\alpha \beta} K_{\alpha \beta}= & -u_{2}^{2} F^{22, r s} u_{r s \alpha \beta} a^{\alpha \beta}-2 u_{2} F^{i 2} u_{i \alpha \beta} a^{\alpha \beta}+3 u_{2} F^{22} u_{2 \alpha \beta} a^{\alpha \beta} \\
& -4 u_{2} F^{i 2, r s} u_{r s \alpha} u_{i \beta} a^{\alpha \beta}+6 u_{2} F^{22, r s} u_{r s \alpha} u_{2 \beta} a^{\alpha \beta} \\
& -2 F^{i j} u_{i \alpha} u_{j \beta} a^{\alpha \beta}+12 F^{i 2} u_{i \alpha} u_{2 \beta} a^{\alpha \beta} \\
& +3 F^{22} u_{k \alpha} u_{k \beta} a^{\alpha \beta}-15 F^{22} u_{2 \alpha} u_{2 \beta} a^{\alpha \beta}
\end{aligned}
$$

By the equation (3.2), we can see

$$
u_{r \alpha \beta} a^{\alpha \beta}=-u_{\alpha \beta}\left(a^{\alpha \beta}\right)_{r}+\left(u_{\alpha \beta} a^{\alpha \beta}\right)_{r}=-u_{\alpha \beta}\left(a^{\alpha \beta}\right)_{r}
$$

and

$$
\begin{aligned}
u_{r s \alpha \beta} a^{\alpha \beta} & =-u_{r \alpha \beta}\left(a^{\alpha \beta}\right)_{s}-u_{s \alpha \beta}\left(a^{\alpha \beta}\right)_{r}-u_{\alpha \beta}\left(a^{\alpha \beta}\right)_{r s}+\left(u_{\alpha \beta} a^{\alpha \beta}\right)_{r s} \\
& =-u_{r \alpha \beta}\left(a^{\alpha \beta}\right)_{s}-u_{s \alpha \beta}\left(a^{\alpha \beta}\right)_{r}-u_{\alpha \beta}\left(a^{\alpha \beta}\right)_{r s} .
\end{aligned}
$$

Hence

$$
\begin{aligned}
u_{2}^{2} F^{22, r s} u_{r s \alpha \beta} a^{\alpha \beta} & =u_{2}^{2} F^{22,11} u_{11 \alpha \beta} a^{\alpha \beta} \\
& =-2 u_{2}^{2} F^{22,11} u_{1 \alpha \beta}\left(a^{\alpha \beta}\right)_{1}-u_{2}^{2} F^{22,11} u_{\alpha \beta}\left(a^{\alpha \beta}\right)_{11}
\end{aligned}
$$

and

$$
u_{2} F^{i 2} u_{i \alpha \beta} a^{\alpha \beta}=-u_{2} F^{i 2} u_{\alpha \beta}\left(a^{\alpha \beta}\right)_{i} .
$$

Substituting the above two equalities into (3.7) shows

$$
\begin{aligned}
|D u|^{3} a^{\alpha \beta} K_{\alpha \beta}= & 2 u_{2}^{2} F^{22,11} u_{1 \alpha \beta}\left(a^{\alpha \beta}\right)_{1}+u_{2}^{2} F^{22,11} u_{\alpha \beta}\left(a^{\alpha \beta}\right)_{11} \\
& +2 u_{2} F^{i 2} u_{\alpha \beta}\left(a^{\alpha \beta}\right)_{i}-3 u_{2} F^{22} u_{\alpha \beta}\left(a^{\alpha \beta}\right)_{2} \\
& -4 u_{2} F^{i 2, r s} u_{r s \alpha} u_{i \beta} a^{\alpha \beta}+6 u_{2} F^{22, r s} u_{r s \alpha} u_{2 \beta} a^{\alpha \beta} \\
& -2 F^{i j} u_{i \alpha} u_{j \beta} a^{\alpha \beta}+12 F^{i 2} u_{i \alpha} u_{2 \beta} a^{\alpha \beta} \\
& +3 F^{22} u_{k \alpha} u_{k \beta} a^{\alpha \beta}-15 F^{22} u_{2 \alpha} u_{2 \beta} a^{\alpha \beta}
\end{aligned}
$$


More precisely we have

$$
|D u|^{3} a^{\alpha \beta} K_{\alpha \beta}:=-[I+I I+I I I],
$$

where

$$
\begin{aligned}
I:= & -2 u_{2}^{2} F^{22,11} u_{1 \alpha \beta}\left(a^{\alpha \beta}\right)_{1}-u_{2}^{2} F^{22,11} u_{\alpha \beta}\left(a^{\alpha \beta}\right)_{11} \\
& -2 u_{2} F^{12} u_{\alpha \beta}\left(a^{\alpha \beta}\right)_{1}+u_{2} F^{22} u_{\alpha \beta}\left(a^{\alpha \beta}\right)_{2}, \\
I I:= & 4 u_{2} F^{12, r s} u_{r s \alpha} u_{1 \beta} a^{\alpha \beta}-2 u_{2} F^{22, r s} u_{r s \alpha} u_{2 \beta} a^{\alpha \beta}, \\
I I I:= & 2 F^{i 1} u_{i \alpha} u_{1 \beta} a^{\alpha \beta}-10 F^{i 2} u_{i \alpha} u_{2 \beta} a^{\alpha \beta} \\
& -3 F^{22} u_{1 \alpha} u_{1 \beta} a^{\alpha \beta}+12 F^{22} u_{2 \alpha} u_{2 \beta} a^{\alpha \beta} .
\end{aligned}
$$

Next, we will calculate the above terms step by step.

Clearly

$$
\left(a^{\alpha \beta}\right)_{1}=2 u_{k} u_{k 1} \delta_{\alpha \beta}+(p-2) u_{1 \alpha} u_{\beta}+(p-2) u_{\alpha} u_{1 \beta}
$$

and

$$
\begin{aligned}
\left(a^{\alpha \beta}\right)_{11}= & 2 u_{k} u_{k 11} \delta_{\alpha \beta}+2 u_{k 1} u_{k 1} \delta_{\alpha \beta} \\
& +(p-2) u_{11 \alpha} u_{\beta}+2(p-2) u_{1 \alpha} u_{1 \beta}+(p-2) u_{\alpha} u_{11 \beta} .
\end{aligned}
$$

Hence we get

$$
\begin{aligned}
I_{1}:= & -2 u_{2}^{2} F^{22,11} u_{1 \alpha \beta}\left(a^{\alpha \beta}\right)_{1} \\
= & -4 u_{2}^{3} u_{1 \alpha \alpha} u_{12}-4(p-2) u_{2}^{3} u_{12 \alpha} u_{1 \alpha} \\
I_{2}:= & -u_{2}^{2} F^{22,11} u_{\alpha \beta}\left(a^{\alpha \beta}\right)_{11} \\
= & -2 u_{2}^{3} u_{112} \triangle u-2 u_{2}^{2} u_{k 1} u_{k 1} \triangle u \\
& \quad-2(p-2) u_{2}^{3} u_{2 \alpha} u_{11 \alpha}-2(p-2) u_{2}^{2} u_{\alpha \beta} u_{1 \alpha} u_{1 \beta}, \\
I_{3}:= & -2 u_{2} F^{12} u_{\alpha \beta}\left(a^{\alpha \beta}\right)_{1} \\
= & 4 u_{2}^{2} u_{12}^{2} \triangle u+4(p-2) u_{2}^{2} u_{12} u_{2 \alpha} u_{1 \alpha}
\end{aligned}
$$

and

$$
\begin{aligned}
I_{4} & :=u_{2} F^{22} u_{\alpha \beta}\left(a^{\alpha \beta}\right)_{2} \\
& =u_{2} u_{11} u_{\alpha \beta}\left(2 u_{k} u_{k 2} \delta_{\alpha \beta}+(p-2) u_{2 \alpha} u_{\beta}+(p-2) u_{\alpha} u_{2 \beta}\right) \\
& =2 u_{2}^{2} u_{11} u_{22} \triangle u+2(p-2) u_{2}^{2} u_{11} u_{2 \alpha} u_{2 \alpha} .
\end{aligned}
$$

Substituting (3.17)-(3.20) into the first term in (3.14), we can see that

$$
\begin{aligned}
I:= & I_{1}+I_{2}+I_{3}+I_{4} \\
= & -2 p u_{2}^{3} u_{12} u_{111}-u_{2}^{3}\left[(4 p-6) u_{11}+2(p-1) u_{22}\right] u_{112} \\
& -4(p-1) u_{2}^{3} u_{12} u_{122}-2(p-1) u_{2}^{2} u_{11}^{3} \\
& +2(p-1) u_{2}^{2} u_{11} u_{12}^{2}+2(p-1) u_{2}^{2} u_{11} u_{22}^{2}+2(p-1) u_{2}^{2} u_{12}^{2} u_{22} .
\end{aligned}
$$

For the second term in (3.14):

$$
\begin{aligned}
I I_{1} & :=4 u_{2} F^{12, r s} u_{r s \alpha} u_{1 \beta} a^{\alpha \beta} \\
& =-4 u_{2}^{3} u_{11} u_{112}-4(p-1) u_{2}^{3} u_{12} u_{122}
\end{aligned}
$$

and

$$
\begin{aligned}
I I_{2} & :=-2 u_{2} F^{22, r s} u_{r s \alpha} u_{2 \beta} a^{\alpha \beta} \\
& =-2 u_{2}^{3} u_{12} u_{111}-2(p-1) u_{2}^{3} u_{22} u_{112}
\end{aligned}
$$


i.e.

$$
\begin{aligned}
I I:= & I I_{1}+I I_{2} \\
= & -2 u_{2}^{3} u_{12} u_{111}-u_{2}^{3}\left[4 u_{11}+2(p-1) u_{22}\right] u_{112} \\
& -4(p-1) u_{2}^{3} u_{12} u_{122} .
\end{aligned}
$$

By (3.21) and (3.24) we have

$$
\begin{aligned}
I+I I:= & -2(p+1) u_{2}^{3} u_{12} u_{111}-u_{2}^{3}\left[(4 p-2) u_{11}+4(p-1) u_{22}\right] u_{112} \\
& -8(p-1) u_{2}^{3} u_{12} u_{122} \\
& -2(p-1) u_{2}^{2} u_{11}^{3}+2(p-1) u_{2}^{2} u_{11} u_{12}^{2} \\
& +2(p-1) u_{2}^{2} u_{11} u_{22}^{2}+2(p-1) u_{2}^{2} u_{12}^{2} u_{22} .
\end{aligned}
$$

Next we deal with the last term in (3.14):

$$
\begin{gathered}
I I I_{1}:=2 F^{i 1} u_{i \alpha} u_{1 \beta} a^{\alpha \beta} \\
\quad=2 u_{22} u_{1 \alpha} u_{1 \beta} a^{\alpha \beta}-2 u_{12} u_{2 \alpha} u_{1 \beta} a^{\alpha \beta} \\
\quad=2 u_{2}^{2} u_{11}^{2} u_{22}-2 u_{2}^{2} u_{11} u_{12}^{2}, \\
I I I_{2}:=-10 F^{i 2} u_{i \alpha} u_{2 \beta} a^{\alpha \beta} \\
=10 u_{12} u_{1 \alpha} u_{2 \beta} a^{\alpha \beta}-10 u_{11} u_{2 \alpha} u_{2 \beta} a^{\alpha \beta} \\
=\quad-10(p-1) u_{2}^{2} u_{11} u_{22}^{2}+10(p-1) u_{2}^{2} u_{12}^{2} u_{22}, \\
I I I_{3} \quad:=-3 F^{22} u_{1 \alpha} u_{1 \beta} a^{\alpha \beta} \\
=-3 u_{2}^{2} u_{11}^{3}-3(p-1) u_{2}^{2} u_{11} u_{12}^{2},
\end{gathered}
$$

and

$$
\begin{aligned}
I I I_{4} & :=12 F^{22} u_{2 \alpha} u_{2 \beta} a^{\alpha \beta} \\
& =12 u_{2}^{2} u_{11} u_{12}^{2}+12(p-1) u_{2}^{2} u_{11} u_{22}^{2} .
\end{aligned}
$$

By (3.26) $-(3.29)$ we have

$$
\begin{aligned}
I I I:= & I I I_{1}+I I I_{2}+I I I_{3}+I I I_{4} \\
= & -3 u_{2}^{2} u_{11}^{3}+2 u_{2}^{2} u_{11}^{2} u_{22}-(3 p-13) u_{2}^{2} u_{11} u_{12}^{2} \\
& +2(p-1) u_{2}^{2} u_{11} u_{22}^{2}+10(p-1) u_{2}^{2} u_{12}^{2} u_{22} .
\end{aligned}
$$

Substituting (3.25) and (3.30) into (3.13), it follows that:

$$
\begin{aligned}
|D u|^{3} a^{\alpha \beta} K_{\alpha \beta}:= & -[I+I I+I I I] \\
= & 2(p+1) u_{2}^{3} u_{12} u_{111}+u_{2}^{3}\left[(4 p-2) u_{11}+4(p-1) u_{22}\right] u_{112} \\
& +8(p-1) u_{2}^{3} u_{12} u_{122} \\
& +(2 p+1) u_{2}^{2} u_{11}^{3}-2 u_{2}^{2} u_{11}^{2} u_{22}+(p-11) u_{2}^{2} u_{11} u_{12}^{2} \\
& -4(p-1) u_{2}^{2} u_{11} u_{22}^{2}-12(p-1) u_{2}^{2} u_{12}^{2} u_{22} .
\end{aligned}
$$

To get (3.3) from (3.31), we must deal with the third derivatives of $u$. Now, by (3.5) we can see

$$
\begin{aligned}
|D u|^{3} K_{\alpha} & =-F^{22, r s} u_{r s \alpha} u_{2}^{2}-2 F^{i 2} u_{i \alpha} u_{2}+3 F^{22} u_{2} u_{2 \alpha} \\
& =-u_{11 \alpha} u_{2}^{2}+2 u_{12} u_{1 \alpha} u_{2}-2 u_{11} u_{2 \alpha} u_{2}+3 u_{11} u_{2} u_{2 \alpha} .
\end{aligned}
$$

By taking $\alpha=1,2$ in (3.32) we can get respectively:

$$
u_{2} u_{111}=3 u_{11} u_{12}-u_{2}^{2} K_{1}
$$

and

$$
u_{2} u_{112}=u_{11} u_{22}+2 u_{12}^{2}-u_{2}^{2} K_{2} .
$$


On the other hand, differentiating the equation (3.2) shows

$$
\begin{aligned}
0=\left(a^{i j} u_{i j}\right)_{\alpha}= & u_{i j \alpha} a^{i j}+u_{i j}\left(a^{i j}\right)_{\alpha} \\
= & u_{2}^{2} u_{11 \alpha}+(p-1) u_{2}^{2} u_{22 \alpha}+2 u_{2} u_{11} u_{2 \alpha} \\
& +2(p-1) u_{2} u_{22} u_{2 \alpha}+2(p-2) u_{2} u_{12} u_{1 \alpha} .
\end{aligned}
$$

Taking $\alpha=1$ in (3.35), we have, together with (3.33):

$$
(p-1) u_{2} u_{122}=-(2 p+1) u_{11} u_{12}-2(p-1) u_{12} u_{22}+u_{2}^{2} K_{2} .
$$

Substituting (3.33), (3.34) and (3.36) into (3.31) to cancel the third derivative of $u$, we have

$$
\begin{aligned}
|D u|^{3} a^{\alpha \beta} K_{\alpha \beta}+G(D K)= & (2 p+1) u_{2}^{2} u_{11}^{3}+4(p-1) u_{2}^{2} u_{11}^{2} u_{22} \\
& -(p+17) u_{2}^{2} u_{11} u_{12}^{2}-20(p-1) u_{2}^{2} u_{12}^{2} u_{22},
\end{aligned}
$$

where $G(D K)=2(p-3) u_{2}^{4} u_{12} K_{1}+\left[(4 p-2) u_{11}+4(p-1) u_{22}\right] u_{2}^{4} K_{2}$.

By equation (3.2) we also have:

$$
\begin{aligned}
0=a^{i j} u_{i j} & =u_{i j}\left[u_{2}^{2} \delta_{i j}+(p-2) u_{i} u_{j}\right] \\
& =u_{2}^{2} u_{11}+(p-1) u_{2}^{2} u_{22} ;
\end{aligned}
$$

i.e.

$$
u_{11}=-(p-1) u_{22}
$$

Combining this with (3.37), we finally get:

$$
|D u| a^{\alpha \beta} K_{\alpha \beta}+b^{\alpha} K_{\alpha}=-u_{11}\left[(3-2 p) u_{11}^{2}+(p-3) u_{12}^{2}\right],
$$

where $b^{1}=2(p-3) u_{2}^{2} u_{12}, b^{2}=2(2 p-3) u_{2}^{2} u_{11}$.

Since the level lines of $u$ are all convex with respect to the normal $D u$, and $K=-\frac{u_{11} u_{2}^{2}}{|D u|^{3}}$ at the fixed point $x$, we know that $u_{11} \leq 0$. Hence, when $\frac{3}{2} \leq p \leq 3$, (3.40) implies (3.3), and the proof of Theorem 1.2 is complete.

\section{Proof of Theorem 1.3}

We follow the same idea as in the proof of Theorem 1.2 in this section; i.e., we deduce a similar $p$-Laplacian inequality. The only difference between two and three dimensions is in the third derivative terms. Of course, this time the terms with third derivatives of $u$ are more complicated than in the two dimensional case, where only linear terms containing third derivatives of $u$ appear. Now in the three dimensional case, we have to treat the whole expression as a quadratic polynomial in the third derivatives of $u$. Since, in the three dimensional case, we have ten third derivative terms, using the equation and the first derivatives of the Gaussian curvature we can get six conditions on the third derivative terms. So at the end, we shall leave four third derivative terms $\left\{u_{111}, u_{112}, u_{113}, u_{123}\right\}$ in the last formulas (4.76) in this section. In the three dimensional case, these third derivative terms as well as the second derivative terms in the formulas (4.76) carry "good" signs. From this we obtain the key inequality (1.4).

Let $u$ be a $p$-harmonic function as in Theorem 1.3, in particular, its level sets are strictly convex with respect to the normal $D u$. By (2.24), the Gaussian curvature of the level sets of $u$ is

$$
K=F^{i j} u_{i} u_{j}|D u|^{-4},
$$


where we have used the notation as in the last section, in addition that

$$
F^{i j, r s, p q}:=\frac{\partial^{3} F}{\partial u_{i j} \partial u_{r s} \partial u_{p q}} .
$$

However, in this section, the repeated indices are summed from 1 to 3 , unless stated otherwise. Now our equation can also be written as:

$$
a^{i j} u_{i j}=0 \text { in } \Omega \text {. }
$$

At any fixed point $x \in \Omega$, we will deduce the following elliptic inequality:

$$
a^{\alpha \beta} K_{\alpha \beta}+b^{\alpha} K_{\alpha} \leq 0
$$

with $b^{\alpha}$ locally bounded. Then by the strong maximum principle [10, we can get our result immediately.

To simplify the calculations, we first rewrite (4.1) as:

$$
|D u|^{4} K=F^{i j} u_{i} u_{j}
$$

Now we begin the proof of Theorem 1.3

Differentiating both sides of (4.5), we get respectively:

$$
\left(|D u|^{4} K\right)_{\alpha}=|D u|^{4} K_{\alpha}+\left(|D u|^{4}\right)_{\alpha} K
$$

and

$$
\left(F^{i j} u_{i} u_{j}\right)_{\alpha}=F^{i j, r s} u_{r s \alpha} u_{i} u_{j}+2 F^{i j} u_{i \alpha} u_{j}
$$

Differentiating respectively once again,

$$
\left(|D u|^{4} K\right)_{\alpha \beta}=|D u|^{4} K_{\alpha \beta}+\left(|D u|^{4}\right)_{\alpha} K_{\beta}+\left(|D u|^{4}\right)_{\beta} K_{\alpha}+\left(|D u|^{4}\right)_{\alpha \beta} K
$$

and

$$
\begin{aligned}
\left(F^{i j} u_{i} u_{j}\right)_{\alpha \beta}= & F^{i j, r s, p q} u_{p q \beta} u_{r s \alpha} u_{i} u_{j}+F^{i j, r s} u_{r s \alpha \beta} u_{i} u_{j}+2 F^{i j, r s} u_{r s \alpha} u_{i \beta} u_{j} \\
& +2 F^{i j, r s} u_{r s \beta} u_{i \alpha} u_{j}+2 F^{i j} u_{i \alpha \beta} u_{j}+2 F^{i j} u_{i \alpha} u_{j \beta} .
\end{aligned}
$$

These mean:

$$
\begin{aligned}
|D u|^{4} K_{\alpha \beta}= & F^{i j, r s, p q} u_{p q \beta} u_{r s \alpha} u_{i} u_{j}+F^{i j, r s} u_{r s \alpha \beta} u_{i} u_{j} \\
& +2 F^{i j, r s} u_{r s \alpha} u_{i \beta} u_{j}+2 F^{i j, r s} u_{r s \beta} u_{i \alpha} u_{j} \\
& +2 F^{i j} u_{i \alpha \beta} u_{j}+2 F^{i j} u_{i \alpha} u_{j \beta} \\
& -\left(|D u|^{4}\right)_{\alpha} K_{\beta}-\left(|D u|^{4}\right)_{\beta} K_{\alpha}-\left(|D u|^{4}\right)_{\alpha \beta} K .
\end{aligned}
$$

Clearly

$$
\begin{aligned}
\left(|D u|^{4}\right)_{\alpha \beta} & =\left(4|D u|^{2} u_{k} u_{k \alpha}\right)_{\beta} \\
& =8 u_{k} u_{k \alpha} u_{l} u_{l \beta}+4|D u|^{2} u_{k \alpha} u_{k \beta}+4|D u|^{2} u_{k} u_{k \alpha \beta} .
\end{aligned}
$$

Substitute (4.11) into (4.10) and multiply both sides by $a^{\alpha \beta}$ to derive:

$$
\begin{aligned}
|D u|^{4} a^{\alpha \beta} K_{\alpha \beta}= & F^{i j, r s, p q} u_{p q \beta} u_{r s \alpha} u_{i} u_{j} a^{\alpha \beta}+F^{i j, r s} u_{r s \alpha \beta} u_{i} u_{j} a^{\alpha \beta} \\
& +4 F^{i j, r s} u_{r s \alpha} u_{i \beta} u_{j} a^{\alpha \beta}+2 F^{i j} u_{i \alpha \beta} u_{j} a^{\alpha \beta} \\
& +2 F^{i j} u_{i \alpha} u_{j \beta} a^{\alpha \beta} \\
& -8 K u_{k} u_{k \alpha} u_{l} u_{l \beta} a^{\alpha \beta}-4 K|D u|^{2} u_{k \alpha} u_{k \beta} a^{\alpha \beta} \\
& -4 K|D u|^{2} u_{k} u_{k \alpha \beta} a^{\alpha \beta}-2 K_{\alpha}\left(|D u|^{4}\right)_{\beta} a^{\alpha \beta} .
\end{aligned}
$$

In order to prove (4.4) at an arbitrary point $x \in \Omega$, as in Caffarelli-Friedman [3, we choose the normal coordinate at $x$. By rotating the coordinate system suitably by $T_{x}$, we may assume that $u_{i}(x)=0,1 \leq i \leq 2$ and $u_{3}(x)=|\nabla u|>0$. We can further assume that the matrix $\left(u_{i j}(x)\right)(1 \leq i, j \leq 2)$ is diagonal and 
$u_{i i}(x)<0,1 \leq i \leq 2$. We also choose $T_{x}$ to vary smoothly with $x$. If we can establish (4.4) at $x$ under the above assumptions, then going back to the original coordinate, we find that (4.4) remains valid with new locally bounded coefficients on $\nabla K$ in (4.4), depending smoothly on the independent variables. Thus it suffices to establish (4.4) under the above assumptions.

From now on, all the calculations will be done at the fixed point $x$; we have $K=F^{33} u_{3} u_{3}|D u|^{-4}=u_{11} u_{22}|D u|^{-2}>0$. Now (4.12) can be rewritten as:

$$
\begin{aligned}
|D u|^{4} a^{\alpha \beta} K_{\alpha \beta}+G_{1}(D K)= & u_{3}^{2} F^{33, r s, p q} u_{p q \beta} u_{r s \alpha} a^{\alpha \beta}+u_{3}^{2} F^{33, r s} u_{r s \alpha \beta} a^{\alpha \beta} \\
& +4 u_{3} F^{i 3, r s} u_{r s \alpha} u_{i \beta} a^{\alpha \beta}+2 u_{3} F^{i 3} u_{i \alpha \beta} a^{\alpha \beta} \\
& +2 F^{i j} u_{i \alpha} u_{j \beta} a^{\alpha \beta}-8 F^{33} u_{3 \alpha} u_{3 \beta} a^{\alpha \beta} \\
& -4 F^{33} u_{k \alpha} u_{k \beta} a^{\alpha \beta}-4 u_{3} F^{33} u_{3 \alpha \beta} a^{\alpha \beta}
\end{aligned}
$$

where $G_{1}(D K)=2\left(|D u|^{4}\right)_{\beta} a^{\alpha \beta} K_{\alpha}$.

Inserting (3.8)-(3.9) into (4.13), it follows that

$$
\begin{aligned}
|D u|^{4} a^{\alpha \beta} K_{\alpha \beta}+G_{1}(D K)= & u_{3}^{2} F^{33, r s, p q} u_{p q \beta} u_{r s \alpha} a^{\alpha \beta} \\
& -2 u_{3}^{2} F^{33, r s} u_{r \alpha \beta}\left(a^{\alpha \beta}\right)_{s}-u_{3}^{2} F^{33, r s} u_{\alpha \beta}\left(a^{\alpha \beta}\right)_{r s} \\
& +4 u_{3} F^{i 3, r s} u_{r s \alpha} u_{i \beta} a^{\alpha \beta}-2 u_{3} F^{i 3} u_{\alpha \beta}\left(a^{\alpha \beta}\right)_{i} \\
& +2 F^{i j} u_{i \alpha} u_{j \beta} a^{\alpha \beta}-8 F^{33} u_{3 \alpha} u_{3 \beta} a^{\alpha \beta} \\
& -4 F^{33} u_{k \alpha} u_{k \beta} a^{\alpha \beta}+4 u_{3} F^{33} u_{\alpha \beta}\left(a^{\alpha \beta}\right)_{3} .
\end{aligned}
$$

The following calculations are easy:

$$
\left(a^{\alpha \beta}\right)_{r}=2 u_{k} u_{k r} \delta_{\alpha \beta}+(p-2) u_{r \alpha} u_{\beta}+(p-2) u_{\alpha} u_{r \beta}
$$

and

$$
\begin{aligned}
\left(a^{\alpha \beta}\right)_{r s}= & 2 u_{k} u_{k r s} \delta_{\alpha \beta}+2 u_{k r} u_{k s} \delta_{\alpha \beta}+(p-2) u_{r s \alpha} u_{\beta} \\
& +(p-2) u_{r \alpha} u_{s \beta}+(p-2) u_{s \alpha} u_{r \beta}+(p-2) u_{\alpha} u_{r s \beta} .
\end{aligned}
$$

Substituting (4.16) into (4.14) we have:

$$
\begin{aligned}
|D u|^{4} a^{\alpha \beta} K_{\alpha \beta}+G_{1}(D k)= & u_{3}^{2} F^{33, r s, p q} u_{p q \beta} u_{r s \alpha} a^{\alpha \beta}-2 u_{3}^{2} F^{33, r s} u_{r \alpha \beta}\left(a^{\alpha \beta}\right)_{s} \\
& -2 u_{3}^{3} F^{33, r s} u_{3 r s} \Delta u-2(p-2) u_{3}^{3} F^{33, r s} u_{r s \alpha} u_{3 \alpha} \\
& +4 u_{3} F^{i 3, r s} u_{r s \alpha} u_{i \beta} a^{\alpha \beta}-2(p-2) u_{3}^{2} F^{33, r s} u_{r \alpha} u_{s \beta} u_{\alpha \beta} \\
& -2 u_{3}^{2} F^{33, r s} u_{k r} u_{k s} \Delta u-2 u_{3} F^{i 3} u_{\alpha \beta}\left(a^{\alpha \beta}\right)_{i} \\
& +2 F^{i j} u_{i \alpha} u_{j \beta} a^{\alpha \beta}-8 F^{33} u_{3 \alpha} u_{3 \beta} a^{\alpha \beta} \\
& -4 F^{33} u_{k \alpha} u_{k \beta} a^{\alpha \beta}+4 u_{3} F^{33} u_{\alpha \beta}\left(a^{\alpha \beta}\right)_{3} \\
:= & u_{3}^{2}(I+I I+I I I),
\end{aligned}
$$

where

$$
\begin{gathered}
I:=F^{33, r s, p q} u_{p q \beta} u_{r s \alpha} a^{\alpha \beta} \\
I I:=-2 F^{33, r s} u_{r \alpha \beta}\left(a^{\alpha \beta}\right)_{s}-2 u_{3} F^{33, r s} u_{3 r s} \Delta u \\
-2(p-2) u_{3} F^{33, r s} u_{r s \alpha} u_{3 \alpha}+\frac{4}{u_{3}} F^{i 3, r s} u_{r s \alpha} u_{i \beta} a^{\alpha \beta}
\end{gathered}
$$


and

$$
\begin{aligned}
I I I:= & -2(p-2) F^{33, r s} u_{r \alpha} u_{s \beta} u_{\alpha \beta}-2 F^{33, r s} u_{k r} u_{k s} \Delta u \\
& -\frac{2}{u_{3}} F^{i 3} u_{\alpha \beta}\left(a^{\alpha \beta}\right)_{i}+\frac{4}{u_{3}} F^{33} u_{\alpha \beta}\left(a^{\alpha \beta}\right)_{3} \\
& +\frac{2}{u_{3}^{2}} F^{i j} u_{i \alpha} u_{j \beta} a^{\alpha \beta}-\frac{8}{u_{3}^{2}} F^{33} u_{3 \alpha} u_{3 \beta} a^{\alpha \beta}-\frac{4}{u_{3}^{2}} F^{33} u_{k \alpha} u_{k \beta} a^{\alpha \beta} .
\end{aligned}
$$

Here we consider the expression as a quadratic polynomial in the third derivatives of $u$ and we group all terms according to the degrees of the third derivatives of $u$.

Next, we calculate all the terms on the right of (4.17) in more detail.

To deal with the third derivatives of $u$, we need some preparations to find the relationship between them.

By (4.6) and (4.7) we have:

$$
\begin{aligned}
|D u|^{4} K_{\alpha}= & -\left(|D u|^{4}\right)_{\alpha} K+F^{i j, r s} u_{r s \alpha} u_{i} u_{j}+2 F^{i j} u_{i \alpha} u_{j} \\
= & -4|D u|^{2} u_{k} u_{k \alpha} K+F^{i j, r s} u_{r s \alpha} u_{i} u_{j}+2 F^{i j} u_{i \alpha} u_{j} \\
= & -4 u_{3} F^{33} u_{3 \alpha}+u_{3}^{2} F^{33, r s} u_{r s \alpha}+2 u_{3} F^{i 3} u_{i \alpha} \\
= & u_{3}^{2} u_{22} u_{11 \alpha}+u_{3}^{2} u_{11} u_{22 \alpha} \\
& -2 u_{3} u_{11} u_{22} u_{3 \alpha}-2 u_{3} u_{11} u_{32} u_{2 \alpha}-2 u_{3} u_{22} u_{31} u_{1 \alpha}
\end{aligned}
$$

i.e.

$$
\begin{aligned}
u_{3}^{2} u_{11} u_{22 \alpha}= & -u_{3}^{2} u_{22} u_{11 \alpha}+2 u_{3} u_{11} u_{22} u_{3 \alpha} \\
& +2 u_{3} u_{11} u_{32} u_{2 \alpha}+2 u_{3} u_{22} u_{31} u_{1 \alpha}+|D u|^{4} K_{\alpha} .
\end{aligned}
$$

Taking $\alpha=1,2,3$ in (4.22) respectively, we can get:

$$
\left\{\begin{array}{l}
u_{3} u_{122}=-\frac{u_{22}}{u_{11}} u_{3} u_{111}+4 u_{13} u_{22}+\frac{u_{3}^{3}}{u_{11}} K_{1}, \\
u_{3} u_{222}=-\frac{u_{22}}{u_{11}} u_{3} u_{112}+4 u_{22} u_{23}+\frac{u_{3}^{3}}{u_{11}} K_{2}, \\
u_{3} u_{223}=-\frac{u_{22}}{u_{11}} u_{3} u_{113}+2 \frac{u_{22}}{u_{11}} u_{13}^{2}+2 u_{22} u_{33}+2 u_{23}^{2}+\frac{u_{3}^{3}}{u_{11}} K_{3} .
\end{array}\right.
$$

We have, moreover, by differentiating equation (4.3):

$$
\begin{aligned}
0=\left(a^{i j} u_{i j}\right)_{\alpha}= & u_{3}^{2} u_{11 \alpha}+u_{3}^{2} u_{22 \alpha}+(p-1) u_{3}^{2} u_{33 \alpha} \\
& +2 u_{3} u_{3 \alpha} \Delta u+2(p-2) u_{3} u_{i 3} u_{i \alpha} ;
\end{aligned}
$$

i.e.

$$
(1-p) u_{3} u_{33 \alpha}=u_{3} u_{11 \alpha}+u_{3} u_{22 \alpha}+2 u_{3 \alpha} \Delta u+2(p-2) u_{i 3} u_{i \alpha} .
$$

Taking $\alpha=1,2$ in (4.25), respectively, and combining (4.23), we can get:

$$
\begin{aligned}
(1-p) u_{3} u_{133}= & u_{3} u_{111}+u_{3} u_{221}+2 u_{31} \Delta u+2(p-2) u_{i 3} u_{i 1} \\
= & \left(1-\frac{u_{22}}{u_{11}}\right) u_{3} u_{111}+2(p-1) u_{11} u_{13}+6 u_{13} u_{22} \\
& +2(p-1) u_{13} u_{33}+\frac{u_{3}^{3}}{u_{11}} K_{1},
\end{aligned}
$$

and

$$
\begin{aligned}
(1-p) u_{3} u_{233}= & u_{3} u_{112}+u_{3} u_{222}+2 u_{32} \Delta u+2(p-2) u_{i 3} u_{i 2} \\
= & \left(1-\frac{u_{22}}{u_{11}}\right) u_{3} u_{112}+2 u_{11} u_{23} \\
& +2(p+1) u_{22} u_{23}+2(p-1) u_{23} u_{33}+\frac{u_{3}^{3}}{u_{11}} K_{2} .
\end{aligned}
$$

Now we are at the position to calculate (4.17) in more detail. First we have:

$$
\begin{aligned}
I:= & F^{33, r s, p q} u_{p q \beta} u_{r s \alpha} a^{\alpha \beta} \\
= & 2 F^{33,22,11} u_{11 \beta} u_{22 \alpha} a^{\alpha \beta}+2 F^{33,12,21} u_{12 \beta} u_{21 \alpha} a^{\alpha \beta} \\
= & 2 u_{3}^{2} u_{111} u_{122}+2 u_{3}^{2} u_{112} u_{222}+2(p-1) u_{3}^{2} u_{113} u_{223} \\
& -2 u_{3}^{2} u_{112}^{2}-2 u_{3}^{2} u_{122}^{2}-2(p-1) u_{3}^{2} u_{123}^{2} .
\end{aligned}
$$


Using (4.23) we get:

$$
\begin{aligned}
2 u_{3}^{2} u_{111} u_{122}-2 u_{3}^{2} u_{122}^{2}= & -2 \frac{u_{22}}{u_{11}}\left(1+\frac{u_{22}}{u_{11}}\right) u_{3}^{2} u_{111}^{2}+8\left(1+2 \frac{u_{22}}{u_{11}}\right) u_{13} u_{22} u_{3} u_{111} \\
& -32 u_{13}^{2} u_{22}^{2} \\
& +\left(2 u_{3}^{4} \frac{u_{11}+2 u_{22}}{u_{11}^{2}} u_{111}-16 u_{3}^{3} \frac{u_{13} u_{22}}{u_{11}}\right) K_{1}-2\left(\frac{u_{3}^{3}}{u_{11}} K_{1}\right)^{2},
\end{aligned}
$$

$$
2 u_{3}^{2} u_{112} u_{222}-2 u_{3}^{2} u_{112}^{2}=-2\left(1+\frac{u_{22}}{u_{11}}\right) u_{3}^{2} u_{112}^{2}+8 u_{22} u_{23} u_{3} u_{112}+2 u_{3}^{4} \frac{u_{112}}{u_{11}} K_{2},
$$

and

$$
\begin{aligned}
2(p-1) u_{3}^{2} u_{113} u_{223}= & -2(p-1) \frac{u_{22}}{u_{11}} u_{3}^{2} u_{113}^{2} \\
& +4(p-1) u_{3} u_{113}\left(\frac{u_{22}}{u_{11}} u_{13}^{2}+u_{22} u_{33}+u_{23}^{2}\right) \\
& +2(p-1) u_{3}^{4} \frac{u_{113}}{u_{11}} K_{3} .
\end{aligned}
$$

Substituting (4.29)-(4.31) into (4.28), it follows that

$$
\begin{aligned}
I:= & F^{33, r s, p q} u_{p q \beta} u_{r s \alpha} a^{\alpha \beta} \\
= & -2 \frac{u_{22}}{u_{11}}\left(1+\frac{u_{22}}{u_{11}}\right) u_{3}^{2} u_{111}^{2}-2\left(1+\frac{u_{22}}{u_{11}}\right) u_{3}^{2} u_{112}^{2} \\
& -2(p-1) \frac{u_{22}}{u_{11}} u_{3}^{2} u_{113}^{2}-2(p-1) u_{3}^{2} u_{123}^{2} \\
& +8\left(1+2 \frac{u_{22}}{u_{11}}\right) u_{13} u_{22} u_{3} u_{111}+8 u_{22} u_{23} u_{3} u_{112} \\
& +4(p-1) u_{3} u_{113}\left(\frac{u_{22}}{u_{11}} u_{13}^{2}+u_{22} u_{33}+u_{23}^{2}\right)-32 u_{13}^{2} u_{22}^{2}+G_{2}(D K)
\end{aligned}
$$

with

$$
\begin{aligned}
G_{2}(D K)= & -2\left(\frac{u_{3}^{3}}{u_{11}} K_{1}\right)^{2}+\left(2 u_{3}^{4} \frac{u_{11}+2 u_{22}}{u_{11}^{2}} u_{111}-16 u_{3}^{3} \frac{u_{13} u_{22}}{u_{11}}\right) K_{1} \\
& +2 u_{3}^{4} \frac{u_{112}}{u_{11}} K_{2}+2(p-1) u_{3}^{4} \frac{u_{113}}{u_{11}} K_{3} .
\end{aligned}
$$

Next we calculate (4.19):

$$
\begin{aligned}
I I_{1}:= & -2 F^{33, r s} u_{r \alpha \beta}\left(a^{\alpha \beta}\right)_{s} \\
= & -2 F^{33,11} u_{1 \alpha \beta}\left(a^{\alpha \beta}\right)_{1}-2 F^{33,22} u_{2 \alpha \beta}\left(a^{\alpha \beta}\right)_{2} \\
= & -4 u_{3} u_{13} u_{22} u_{111}-4 u_{3} u_{11} u_{23} u_{112}-4(p-2) u_{3} u_{22} u_{11} u_{113} \\
& -4 u_{3} u_{13} u_{22} u_{122}-4 u_{3} u_{11} u_{23} u_{222}-4(p-2) u_{3} u_{11} u_{22} u_{223} \\
& -4(p-1) u_{3} u_{13} u_{22} u_{133}-4(p-1) u_{3} u_{11} u_{23} u_{233} .
\end{aligned}
$$

For the terms in the last step of (4.34), we have, by using (4.23):

$$
\begin{aligned}
I I_{11} & :=-4 u_{3} u_{13} u_{22} u_{111}-4 u_{3} u_{13} u_{22} u_{122} \\
& =-4\left(1-\frac{u_{22}}{u_{11}}\right) u_{13} u_{22} u_{3} u_{111}-16 u_{13}^{2} u_{22}^{2}-4 u_{3}^{3} \frac{u_{13} u_{22}}{u_{11}} K_{1}, \\
I I_{12} & :=-4 u_{3} u_{11} u_{23} u_{112}-4 u_{3} u_{11} u_{23} u_{222} \\
& =-4\left(1-\frac{u_{22}}{u_{11}}\right) u_{11} u_{23} u_{3} u_{112}-16 u_{11} u_{22} u_{23}^{2}-4 u_{3}^{3} u_{23} K_{2},
\end{aligned}
$$

and

(4.37)

$$
\begin{aligned}
I I_{13}:= & -4(p-2) u_{3} u_{11} u_{22} u_{113}-4(p-2) u_{3} u_{11} u_{22} u_{223} \\
= & -4(p-2)\left(u_{11} u_{22}-u_{22}^{2}\right) u_{3} u_{113} \\
& -8(p-2)\left(u_{11} u_{22}^{2} u_{33}+u_{11} u_{22} u_{23}^{2}+u_{13}^{2} u_{22}^{2}\right)-4(p-2) u_{3}^{3} u_{22} K_{3} .
\end{aligned}
$$

By (4.26) we also have:

$$
\begin{aligned}
I I_{14}:= & -4(p-1) u_{3} u_{13} u_{22} u_{133} \\
= & 4\left(1-\frac{u_{22}}{u_{11}}\right) u_{13} u_{22} u_{3} u_{111}+8(p-1) u_{11} u_{13}^{2} u_{22} \\
& +24 u_{13}^{2} u_{22}^{2}+8(p-1) u_{13}^{2} u_{22} u_{33}+4 u_{3}^{3} \frac{u_{13} u_{22}}{u_{11}} K_{1} .
\end{aligned}
$$


Similarly we have, by (4.27):

$$
\begin{aligned}
I I_{15}:= & -4(p-1) u_{3} u_{11} u_{23} u_{233} \\
= & 4\left(1-\frac{u_{22}}{u_{11}}\right) u_{11} u_{23} u_{3} u_{112}+8 u_{11}^{2} u_{23}^{2} \\
& +8(p+1) u_{11} u_{22} u_{23}^{2}+8(p-1) u_{11} u_{23}^{2} u_{33}+4 u_{3}^{3} u_{23} K_{2} .
\end{aligned}
$$

Substituting (4.35)-(4.39) into (4.34) we have

$$
\begin{aligned}
I I_{1}:= & -2 F^{33, r s} u_{r \alpha \beta}\left(a^{\alpha \beta}\right)_{s} \\
:= & I I_{11}+I I_{12}+I I_{13}+I I_{14}+I I_{15} \\
= & -4(p-2)\left(u_{11} u_{22}-u_{22}^{2}\right) u_{3} u_{113} \\
& +8 u_{11}^{2} u_{23}^{2}+8(p-1) u_{11} u_{13}^{2} u_{22}-8(p-2) u_{11} u_{22}^{2} u_{33} \\
& +8 u_{11} u_{22} u_{23}^{2}+8(p-1) u_{11} u_{23}^{2} u_{33}-8(p-3) u_{13}^{2} u_{22}^{2} \\
& +8(p-1) u_{13}^{2} u_{22} u_{33}-4(p-2) u_{3}^{3} u_{22} K_{3} .
\end{aligned}
$$

For the second term on the right-hand side of (4.19), again using (4.23) we have:

$$
\begin{aligned}
I I_{2}:= & -2 u_{3} F^{33, r s} u_{3 r s} \Delta u \\
= & -2 u_{3} u_{22} u_{311} \Delta u-2 u_{3} u_{11} u_{322} \Delta u \\
= & -4 u_{11}^{2} u_{22} u_{33}-4 u_{11}^{2} u_{23}^{2}-4 u_{11} u_{13}^{2} u_{22} \\
& -4 u_{11} u_{22}^{2} u_{33}-4 u_{11} u_{22} u_{23}^{2}-4 u_{13}^{2} u_{22}^{2} \\
& -4 u_{11} u_{22} u_{33}^{2}-4 u_{11} u_{23}^{2} u_{33}-4 u_{13}^{2} u_{22} u_{33}-2 u_{3}^{3} \Delta u K_{3} .
\end{aligned}
$$

For the third term on the right-hand side of (4.19), using (4.22) we similarly deduce:

$$
\begin{aligned}
I I_{3}:= & -2(p-2) u_{3} F^{33, r s} u_{r s \alpha} u_{3 \alpha} \\
= & -2(p-2) u_{3} u_{22} u_{11 \alpha} u_{3 \alpha}-2(p-2) u_{3} u_{11} u_{22 \alpha} u_{3 \alpha} \\
= & -8(p-2) u_{11} u_{13}^{2} u_{22}-8(p-2) u_{11} u_{22} u_{23}^{2} \\
& -4(p-2) u_{13}^{2} u_{22} u_{33}-4(p-2) u_{11} u_{22} u_{33}^{2}-4(p-2) u_{11} u_{23}^{2} u_{33} \\
& -2(p-2) u_{3}^{3} u_{3 \alpha} K_{\alpha} .
\end{aligned}
$$

The fourth term on the right-hand side of (4.19) is:

$$
\begin{aligned}
I I_{4}:= & \frac{4}{u_{3}} F^{i 3, r s} u_{r s \alpha} u_{i \beta} a^{\alpha \beta} \\
= & \frac{4}{u_{3}} F^{13, r s} u_{r s \alpha} u_{1 \beta} a^{\alpha \beta}+\frac{4}{u_{3}} F^{23, r s} u_{r s \alpha} u_{2 \beta} a^{\alpha \beta} \\
& +\frac{4}{u_{3}} F^{33, r s} u_{r s \alpha} u_{3 \beta} a^{\alpha \beta} .
\end{aligned}
$$

We will calculate the three terms on the right-hand side of (4.43) respectively:

$$
\begin{aligned}
I I_{41}:= & \frac{4}{u_{3}} F^{13, r s} u_{r s \alpha} u_{1 \beta} a^{\alpha \beta} \\
= & \frac{4}{u_{3}} F^{13,31} u_{31 \alpha} u_{1 \beta} a^{\alpha \beta}+\frac{4}{u_{3}} F^{13,22} u_{22 \alpha} u_{1 \beta} a^{\alpha \beta} \\
& +\frac{4}{u_{3}} F^{13,21} u_{21 \alpha} u_{1 \beta} a^{\alpha \beta} \\
= & 4 u_{11} u_{23} u_{3} u_{112}-4 u_{11} u_{13} u_{3} u_{122}-4 u_{11} u_{22} u_{3} u_{113} \\
& -4(p-1) u_{13}^{2} u_{3} u_{223}-4(p-1) u_{13} u_{22} u_{3} u_{133} \\
& +4(p-1) u_{13} u_{23} u_{3} u_{123} .
\end{aligned}
$$

By (4.23) we have:

$$
-4 u_{11} u_{13} u_{3} u_{122}=4 u_{13} u_{22} u_{3} u_{111}-16 u_{11} u_{13}^{2} u_{22}-4 u_{3}^{3} u_{13} K_{1},
$$


and

$$
\begin{aligned}
-4 u_{11} u_{22} u_{3} u_{113}-4(p-1) u_{13}^{2} u_{3} u_{223} \\
=\left[-4 u_{11} u_{22}+4(p-1) \frac{u_{22}}{u_{11}} u_{13}^{2}\right] u_{3} u_{113} \\
\quad-8(p-1)\left(\frac{u_{22}}{u_{11}} u_{13}^{4}+u_{13}^{2} u_{22} u_{33}+u_{13}^{2} u_{23}^{2}\right) \\
\quad-4(p-1) u_{3}^{3} \frac{u_{13}^{2}}{u_{11}} K_{3} .
\end{aligned}
$$

By (4.26) we see:

$$
\begin{aligned}
& -4(p-1) u_{13} u_{22} u_{3} u_{133} \\
= & 4\left(1-\frac{u_{22}}{u_{11}}\right) u_{13} u_{22} u_{3} u_{111} \\
& +8(p-1) u_{11} u_{13}^{2} u_{22}+24 u_{13}^{2} u_{22}^{2}+8(p-1) u_{13}^{2} u_{22} u_{33}+4 u_{3}^{3} \frac{u_{13} u_{22}}{u_{11}} K_{1} .
\end{aligned}
$$

Substituting (4.45)-(4.47) into (4.44), it follows that

$$
\begin{aligned}
I I_{41}:= & \frac{4}{u_{3}} F^{13, r s} u_{r s \alpha} u_{1 \beta} a^{\alpha \beta} \\
= & 4\left(2-\frac{u_{22}}{u_{11}}\right) u_{13} u_{22} u_{3} u_{111}+4 u_{11} u_{23} u_{3} u_{112} \\
& -4 \frac{u_{22}}{u_{11}}\left[u_{11}^{2}-(p-1) u_{13}^{2}\right] u_{3} u_{113} \\
& +4(p-1) u_{13} u_{23} u_{3} u_{123}+8(p-3) u_{11} u_{13}^{2} u_{22} \\
& -8(p-1) \frac{u_{22}}{u_{11}} u_{13}^{4}+24 u_{13}^{2} u_{22}^{2}-8(p-1) u_{13}^{2} u_{23}^{2} \\
& +4 u_{3}^{3} \frac{u_{22}-u_{11}}{u_{11}} u_{13} K_{1}-4(p-1) u_{3}^{3} \frac{u_{13}^{2}}{u_{11}} K_{3} .
\end{aligned}
$$

For the second term on the right-hand side of (4.43):

$$
\begin{aligned}
I I_{42}:= & \frac{4}{u_{3}} F^{23, r s} u_{r s \alpha} u_{2 \beta} a^{\alpha \beta} \\
= & \frac{4}{u_{3}} F^{23,32} u_{32 \alpha} u_{2 \beta} a^{\alpha \beta}+\frac{4}{u_{3}} F^{23,11} u_{11 \alpha} u_{2 \beta} a^{\alpha \beta} \\
& +\frac{4}{u_{3}} F^{23,12} u_{12 \alpha} u_{2 \beta} a^{\alpha \beta} \\
= & -4 u_{11} u_{22} u_{3} u_{223}-4(p-1) u_{11} u_{23} u_{3} u_{233} \\
& -4 u_{22} u_{23} u_{3} u_{112}-4(p-1) u_{23}^{2} u_{3} u_{113} \\
& +4 u_{13} u_{22} u_{3} u_{122}+4(p-1) u_{13} u_{23} u_{3} u_{123} .
\end{aligned}
$$

Also using (4.23) yields:

$$
4 u_{13} u_{22} u_{3} u_{122}=-4 \frac{u_{22}}{u_{11}} u_{13} u_{22} u_{3} u_{111}+16 u_{13}^{2} u_{22}^{2}+4 u_{3}^{3} \frac{u_{13} u_{22}}{u_{11}} K_{1}
$$

and

$$
\begin{aligned}
& -4(p-1) u_{23}^{2} u_{3} u_{113}-4 u_{11} u_{22} u_{3} u_{223} \\
= & {\left[4 u_{22}^{2}-4(p-1) u_{23}^{2}\right] u_{3} u_{113} } \\
& -8 u_{13}^{2} u_{22}^{2}-8 u_{11} u_{22}^{2} u_{33}-8 u_{11} u_{22} u_{23}^{2}-4 u_{3}^{3} u_{22} K_{3} .
\end{aligned}
$$

By (4.27):

$$
\begin{aligned}
& -4 u_{22} u_{23} u_{3} u_{112}-4(p-1) u_{11} u_{23} u_{3} u_{233} \\
= & 4\left(1-2 \frac{u_{22}}{u_{11}}\right) u_{11} u_{23} u_{3} u_{112} \\
& +8 u_{11}^{2} u_{23}^{2}+8(p+1) u_{11} u_{22} u_{23}^{2}+8(p-1) u_{11} u_{23}^{2} u_{33}+4 u_{3}^{3} u_{23} K_{2} .
\end{aligned}
$$


Substituting (4.50)-(4.52) into (4.49) yields:

$$
\begin{aligned}
I I_{42}:= & \frac{4}{u_{3}} F^{23, r s} u_{r s \alpha} u_{2 \beta} a^{\alpha \beta} \\
= & -4 \frac{u_{22}}{u_{11}} u_{13} u_{22} u_{3} u_{111}+4\left(1-2 \frac{u_{22}}{u_{11}}\right) u_{11} u_{23} u_{3} u_{112} \\
& +\left[4 u_{22}^{2}-4(p-1) u_{23}^{2}\right] u_{3} u_{113}+4(p-1) u_{13} u_{23} u_{3} u_{123} \\
& +8 u_{11}^{2} u_{23}^{2}-8 u_{11} u_{22}^{2} u_{33}+8 p u_{11} u_{22} u_{23}^{2} \\
& +8(p-1) u_{11} u_{23}^{2} u_{33}+8 u_{13}^{2} u_{22}^{2} \\
& +4 u_{3}^{3} \frac{u_{13} u_{22}}{u_{11}} K_{1}+4 u_{3}^{3} u_{23} K_{2}-4 u_{3}^{3} u_{22} K_{3} .
\end{aligned}
$$

For the third term on the right-hand side of (4.43), using (4.22) we have:

$$
\begin{aligned}
I I_{43}:= & \frac{4}{u_{3}} F^{33, r s} u_{r s \alpha} u_{3 \beta} a^{\alpha \beta} \\
= & \frac{4}{u_{3}} u_{22} u_{11 \alpha} u_{3 \beta} a^{\alpha \beta}+\frac{4}{u_{3}} u_{11} u_{22 \alpha} u_{3 \beta} a^{\alpha \beta} \\
= & 16 u_{11} u_{22} u_{13}^{2}+16 u_{11} u_{22} u_{23}^{2} \\
& +8(p-1)\left(u_{11} u_{22} u_{33}^{2}+u_{11} u_{23}^{2} u_{33}+u_{13}^{2} u_{22} u_{33}\right)+4 u_{3} a^{\alpha \beta} u_{3 \beta} K_{\alpha} .
\end{aligned}
$$

Substituting (4.48) and (4.53)-(4.54) into (4.43) yields:

$$
\begin{aligned}
I I_{4}= & 8\left(1-\frac{u_{22}}{u_{11}}\right) u_{13} u_{22} u_{3} u_{111}+8\left(1-\frac{u_{22}}{u_{11}}\right) u_{11} u_{23} u_{3} u_{112}+8(p-1) u_{13} u_{23} u_{3} u_{123} \\
& +\left[4 u_{22}^{2}-4 u_{11} u_{22}-4(p-1) u_{23}^{2}+4(p-1) \frac{u_{22}}{u_{11}} u_{13}^{2}\right] u_{3} u_{113} \\
& +8 u_{11}^{2} u_{23}^{2}+8(p-1) u_{11} u_{13}^{2} u_{22}-8 u_{11} u_{22}^{2} u_{33}+8(p+2) u_{11} u_{22} u_{23}^{2} \\
& +8(p-1) u_{11} u_{22} u_{33}^{2}+16(p-1) u_{11} u_{23}^{2} u_{33}-8(p-1) \frac{u_{22}}{u_{11}} u_{13}^{4} \\
& +32 u_{13}^{2} u_{22}^{2}+8(p-1) u_{13}^{2} u_{22} u_{33}-8(p-1) u_{13}^{2} u_{23}^{2} \\
& +8 u_{3}^{3} \frac{u_{13} u_{22}}{u_{11}} K_{1}+8 u_{3}^{3} u_{23} K_{2}-4 u_{3}^{3} u_{22} K_{3}-4(p-1) u_{3}^{3}\left(\frac{u_{13}^{2}}{u_{11}}-u_{33}\right) K_{3} .
\end{aligned}
$$

Substituting (4.40)-(4.42) and (4.55) into (4.19), it follows that

$$
\begin{aligned}
I I:= & -2 F^{33, r s} u_{r \alpha \beta}\left(a^{\alpha \beta}\right)_{s}-2 u_{3} F^{33, r s} u_{3 r s} \Delta u \\
& -2(p-2) u_{3} F^{33, r s} u_{r s \alpha} u_{3 \alpha}+\frac{4}{u_{3}} F^{i 3, r s} u_{r s \alpha} u_{i \beta} a^{\alpha \beta} \\
:= & I I_{1}+I I_{2}+I I_{3}+I I_{4} \\
= & 8\left(1-\frac{u_{22}}{u_{11}}\right) u_{13} u_{22} u_{3} u_{111}+8\left(1-\frac{u_{22}}{u_{11}}\right) u_{11} u_{23} u_{3} u_{112}+8(p-1) u_{13} u_{23} u_{3} u_{123} \\
& +\left[4(p-1) u_{22}^{2}-4(p-1) u_{11} u_{22}-4(p-1) u_{23}^{2}+4(p-1) \frac{u_{22}}{u_{11}} u_{13}^{2}\right] u_{3} u_{113} \\
& -4 u_{11}^{2} u_{22} u_{33}+12 u_{11}^{2} u_{23}^{2}+(8 p-4) u_{11} u_{13}^{2} u_{22}-(8 p-4) u_{11} u_{22}^{2} u_{33} \\
& +36 u_{11} u_{22} u_{23}^{2}+4(p-1) u_{11} u_{22} u_{33}^{2}+20(p-1) u_{11} u_{23}^{2} u_{33} \\
& -(8 p-52) u_{13}^{2} u_{22}^{2}+12(p-1) u_{13}^{2} u_{22} u_{33}-8(p-1) u_{13}^{2} u_{23}^{2} \\
& -8(p-1) \frac{u_{22}}{u_{11}} u_{13}^{4}+G_{3}(D K)
\end{aligned}
$$


with

$$
\begin{aligned}
G_{3}(D K)= & \left(8 \frac{u_{22}}{u_{11}}-2 p+4\right) u_{3}^{3} u_{13} K_{1}-2(p-6) u_{3}^{3} u_{23} K_{2}+(6 p-8) u_{3}^{3} u_{33} K_{3} \\
& -4(p-1) u_{3}^{3} \frac{u_{11} u_{22}+u_{13}^{2}}{u_{11}} K_{3},
\end{aligned}
$$

where we have used $\Delta u=-(p-2) u_{33}$ by equation (4.3).

Next we calculate the third term (4.20):

$$
\begin{aligned}
I I I:= & -2(p-2) F^{33, r s} u_{r \alpha} u_{s \beta} u_{\alpha \beta}-2 F^{33, r s} u_{k r} u_{k s} \Delta u \\
& -\frac{2}{u_{3}} F^{13} u_{\alpha \beta}\left(a^{\alpha \beta}\right)_{1}-\frac{2}{u_{3}} F^{23} u_{\alpha \beta}\left(a^{\alpha \beta}\right)_{2}+\frac{2}{u_{3}} F^{33} u_{\alpha \beta}\left(a^{\alpha \beta}\right)_{3} \\
& +\frac{2}{u_{3}^{2}} F^{i j} u_{i \alpha} u_{j \beta} a^{\alpha \beta}-\frac{8}{u_{3}^{2}} F^{33} u_{3 \alpha} u_{3 \beta} a^{\alpha \beta}-\frac{4}{u_{3}^{2}} F^{33} u_{k \alpha} u_{k \beta} a^{\alpha \beta} \\
:= & I I I_{1}+I I I_{2}+I I I_{3} .
\end{aligned}
$$

We shall deal with $I I I_{1}, I I I_{2}$ and $I I I_{3}$ respectively as follows:

$$
\begin{aligned}
I I I_{1}:= & -2(p-2) F^{33, r s} u_{r \alpha} u_{s \beta} u_{\alpha \beta}-2 F^{33, r s} u_{k r} u_{k s} \Delta u \\
= & -2(p-2) u_{11} u_{2 \alpha} u_{2 \beta} u_{\alpha \beta}-2(p-2) u_{22} u_{1 \alpha} u_{1 \beta} u_{\alpha \beta} \\
& -2 u_{11} u_{k 2} u_{k 2} \Delta u-2 u_{22} u_{k 1} u_{k 1} \Delta u \\
= & -2(p-1) u_{11}^{3} u_{22}-4 u_{11}^{2} u_{22}^{2}-2 u_{11}^{2} u_{22} u_{33}-2 u_{11}^{2} u_{23}^{2} \\
& +(6-4 p)\left(u_{11} u_{13}^{2} u_{22}+u_{11} u_{22} u_{23}^{2}\right)-2(p-1) u_{11} u_{22}^{3}-2 u_{11} u_{22}^{2} u_{33} \\
- & 2(p-1) u_{11} u_{23}^{2} u_{33}-2 u_{13}^{2} u_{22}^{2}-2(p-1) u_{13}^{2} u_{22} u_{33}, \\
I I I_{2}:= & -\frac{2}{u_{3}} F^{13} u_{\alpha \beta}\left(a^{\alpha \beta}\right)_{1}-\frac{2}{u_{3}} F^{23} u_{\alpha \beta}\left(a^{\alpha \beta}\right)_{2}+\frac{2}{u_{3}} F^{33} u_{\alpha \beta}\left(a^{\alpha \beta}\right)_{3} \\
= & 4 u_{11}^{2} u_{22} u_{33}+(8 p-12) u_{11} u_{13}^{2} u_{22}+4 u_{11}^{2} u_{23}^{2} \\
& +4 u_{11} u_{22}^{2} u_{33}+(8 p-12) u_{11} u_{22} u_{23}^{2}+4(p-1) u_{11} u_{22} u_{33}^{2} \\
& +4(p-1) u_{11} u_{23}^{2} u_{33}+4(p-1) u_{13}^{2} u_{22} u_{33}+4 u_{13}^{2} u_{22}^{2}, \\
\text {. } & \\
I I I_{3}:= & \frac{2}{u_{3}^{2}} F^{i j} u_{i \alpha} u_{j \beta} a^{\alpha \beta}-\frac{8}{u_{3}^{2}} F^{33} u_{3 \alpha} u_{3 \beta} a^{\alpha \beta}-\frac{4}{u_{3}^{2}} F^{33} u_{k \alpha} u_{k \beta} a^{\alpha \beta} \\
= & \frac{2}{u_{3}^{2}} F^{i j} u_{i \alpha} u_{j \beta} a^{\alpha \beta} \\
& -\frac{12}{u_{3}^{2}} F^{33} u_{3 \alpha} u_{3 \beta} a^{\alpha \beta}-\frac{4}{u_{3}^{2}} F^{33} u_{1 \alpha} u_{1 \beta} a^{\alpha \beta}-\frac{4}{u_{3}^{2}} F^{33} u_{2 \alpha} u_{2 \beta} a^{\alpha \beta} \\
:= & I I I_{31}+I I I_{32}+I I I_{33},
\end{aligned}
$$

where

$$
\begin{aligned}
I I I_{31}:= & \frac{2}{u_{3}^{2}} F^{i j} u_{i \alpha} u_{j \beta} a^{\alpha \beta} \\
= & \frac{2}{u_{3}^{2}} F^{11} u_{1 \alpha} u_{1 \beta} a^{\alpha \beta}+\frac{2}{u_{3}^{2}} F^{22} u_{2 \alpha} u_{2 \beta} a^{\alpha \beta}+\frac{2}{u_{3}^{2}} F^{33} u_{3 \alpha} u_{3 \beta} a^{\alpha \beta} \\
& +\frac{4}{u_{3}^{2}} F^{12} u_{1 \alpha} u_{2 \beta} a^{\alpha \beta}+\frac{4}{u_{3}^{2}} F^{13} u_{1 \alpha} u_{3 \beta} a^{\alpha \beta}+\frac{4}{u_{3}^{2}} F^{23} u_{2 \alpha} u_{3 \beta} a^{\alpha \beta} .
\end{aligned}
$$


The six terms in (4.61) are precisely:

$$
\begin{aligned}
& \frac{2}{u_{3}^{3}} F^{11} u_{1 \alpha} u_{1 \beta} a^{\alpha \beta}=\frac{2}{u_{3}^{3}}\left(u_{22} u_{33}-u_{23} u_{32}\right) u_{1 \alpha} u_{1 \beta}\left[u_{3}^{2} \delta_{\alpha \beta}+(p-2) u_{\alpha} u_{\beta}\right] \\
& =2 u_{11}^{2} u_{22} u_{33}-2 u_{11}^{2} u_{23}^{2}+2(p-1)\left(u_{13}^{2} u_{22} u_{33}-u_{13}^{2} u_{23}^{2}\right) \text {, } \\
& \frac{2}{u_{3}^{2}} F^{22} u_{2 \alpha} u_{2 \beta} a^{\alpha \beta}=\frac{2}{u_{3}^{2}}\left(u_{11} u_{33}-u_{13}^{2}\right) u_{2 \alpha} u_{2 \beta}\left[u_{3}^{2} \delta_{\alpha \beta}+(p-2) u_{\alpha} u_{\beta}\right] \\
& =2 u_{11} u_{22}^{2} u_{33}+2(p-1)\left(u_{11} u_{23}^{2} u_{33}-u_{13}^{2} u_{23}^{2}\right)-2 u_{13}^{2} u_{22}^{2} \text {, } \\
& \frac{2}{u_{3}^{3}} F^{33} u_{3 \alpha} u_{3 \beta} a^{\alpha \beta}=\frac{2}{u_{3}^{2}} u_{11} u_{22} u_{3 \alpha} u_{3 \beta}\left[u_{3}^{2} \delta_{\alpha \beta}+(p-2) u_{\alpha} u_{\beta}\right] \\
& =2 u_{11} u_{13}^{2} u_{22}+2 u_{11} u_{22} u_{23}^{2}+2(p-1) u_{11} u_{22} u_{33}^{2} \text {, } \\
& \frac{4}{u_{3}^{3}} F^{12} u_{1 \alpha} u_{2 \beta} a^{\alpha \beta}=\frac{4}{u_{3}^{2}} u_{31} u_{23} u_{1 \alpha} u_{2 \beta}\left[u_{3}^{2} \delta_{\alpha \beta}+(p-2) u_{\alpha} u_{\beta}\right] \\
& =4(p-1) u_{13}^{2} u_{23}^{2} \text {, } \\
& \frac{4}{u_{3}^{3}} F^{13} u_{1 \alpha} u_{3 \beta} a^{\alpha \beta}=\frac{4}{u_{3}^{3}}\left(-u_{22} u_{31}\right) u_{1 \alpha} u_{3 \beta}\left[u_{3}^{2} \delta_{\alpha \beta}+(p-2) u_{\alpha} u_{\beta}\right] \\
& =-4 u_{11} u_{13}^{2} u_{22}-4(p-1) u_{13}^{2} u_{22} u_{33} \text {, }
\end{aligned}
$$

and

$$
\begin{aligned}
\frac{4}{u_{3}^{3}} F^{23} u_{2 \alpha} u_{3 \beta} a^{\alpha \beta} & =\frac{4}{u_{3}^{2}}\left(-u_{11} u_{32}\right) u_{2 \alpha} u_{3 \beta}\left[u_{3}^{2} \delta_{\alpha \beta}+(p-2) u_{\alpha} u_{\beta}\right] \\
& =-4 u_{11} u_{22} u_{23}^{2}-4(p-1) u_{11} u_{23}^{2} u_{33} .
\end{aligned}
$$

Substituting (4.62)-(4.67) into (4.61), it follows that

$$
\begin{aligned}
I I I_{31}:= & \frac{2}{u_{3}^{2}} F^{i j} u_{i \alpha} u_{j \beta} a^{\alpha \beta} \\
= & 2 u_{11}^{2} u_{22} u_{33}-2 u_{11}^{2} u_{23}^{2}-2 u_{11} u_{13}^{2} u_{22}+2 u_{11} u_{22}^{2} u_{33} \\
& -2 u_{11} u_{22} u_{23}^{2}+2(p-1) u_{11} u_{22} u_{33}^{2}-2(p-1) u_{11} u_{23}^{2} u_{33} \\
& -2(p-1) u_{13}^{2} u_{22} u_{33}-2 u_{13}^{2} u_{22}^{2} .
\end{aligned}
$$

For the second term in (4.60):

$$
\begin{aligned}
I I I_{32} & :=-\frac{12}{u_{3}^{3}} F^{33} u_{3 \alpha} u_{3 \beta} a^{\alpha \beta} \\
& =-12 u_{11} u_{13}^{2} u_{22}-12 u_{11} u_{22} u_{23}^{2}-12(p-1) u_{11} u_{22} u_{33}^{2} .
\end{aligned}
$$

For the last term in (4.60):

$$
\begin{aligned}
I I I_{33}:= & -\frac{4}{u^{3}} F^{33} u_{1 \alpha} u_{1 \beta} a^{\alpha \beta}-\frac{4}{u_{3}^{3}} F^{33} u_{2 \alpha} u_{2 \beta} a^{\alpha \beta} \\
= & -4 u_{11}^{3} u_{22}-4(p-1) u_{11} u_{13}^{2} u_{22} \\
& -4 u_{11} u_{22}^{3}-4(p-1) u_{11} u_{22} u_{23}^{2} .
\end{aligned}
$$

Combining (4.68)-(4.70) with (4.60) yields:

$$
\begin{aligned}
I I I_{3}= & -4 u_{11}^{3} u_{22}+2 u_{11}^{2} u_{22} u_{33}-2 u_{11}^{2} u_{23}^{2}-(4 p+10) u_{11} u_{13}^{2} u_{22} \\
& -4 u_{11} u_{22}^{3}+2 u_{11} u_{22}^{2} u_{33}-(4 p+10) u_{11} u_{22} u_{23}^{2} \\
& -10(p-1) u_{11} u_{22} u_{33}^{2}-2(p-1) u_{11} u_{23}^{2} u_{33} \\
& -2(p-1) u_{13}^{2} u_{22} u_{33}-2 u_{13}^{2} u_{22}^{2} .
\end{aligned}
$$

Substituting (4.58)-(4.59) and (4.71) into (4.57), we have

$$
\begin{aligned}
I I I:= & -2(p-2) F^{33, r s} u_{r \alpha} u_{s \beta} u_{\alpha \beta}-2 F^{33, r s} u_{k r} u_{k s} \Delta u \\
& -\frac{2}{u_{3}} F^{13} u_{\alpha \beta}\left(a^{\alpha \beta}\right)_{1}-\frac{2}{u_{3}} F^{23} u_{\alpha \beta}\left(a^{\alpha \beta}\right)_{2}+2 u_{3} F^{33} u_{\alpha \beta}\left(a^{\alpha \beta}\right)_{3} \\
& +\frac{2}{u_{3}^{3}} F^{i j} u_{i \alpha} u_{j \beta} a^{\alpha \beta}-\frac{8}{u_{3}^{3}} F^{33} u_{3 \alpha} u_{3 \beta} a^{\alpha \beta}-\frac{4}{u_{3}^{3}} F^{33} u_{k \alpha} u_{k \beta} a^{\alpha \beta} \\
:= & I I I_{1}+I I I_{2}+I I I_{3} \\
= & -2(p+1) u_{11}^{3} u_{22}-4 u_{11}^{2} u_{22}^{2}+4 u_{11}^{2} u_{22} u_{33}-16 u_{11} u_{13}^{2} u_{22} \\
& -2(p+1) u_{11} u_{22}^{3}+4 u_{11} u_{22}^{2} u_{33}-16 u_{11} u_{22} u_{23}^{2}-6(p-1) u_{11} u_{22} u_{33}^{2} .
\end{aligned}
$$


Finally, substituting (4.32), (4.56) and (4.72) into (4.17), we get:

$$
\begin{aligned}
|D u|^{2} a^{\alpha \beta} & K_{\alpha \beta}+G(D K) \\
:= & I+I I+I I I \\
= & -2 \frac{u_{22}}{u_{11}}\left(1+\frac{u_{22}}{u_{11}}\right) u_{3}^{2} u_{111}^{2}-2\left(1+\frac{u_{22}}{u_{11}}\right) u_{3}^{2} u_{112}^{2} \\
& -2(p-1) \frac{u_{22}}{u_{11}} u_{3}^{2} u_{113}^{2}-2(p-1) u_{3}^{2} u_{123}^{2} \\
& +8\left(2+\frac{u_{22}}{u_{11}}\right) u_{13} u_{22} u_{3} u_{111}+8 u_{11} u_{23} u_{3} u_{112} \\
& +4(p-1) u_{3} u_{113}\left(2 \frac{u_{22}}{u_{11}} u_{13}^{2}-u_{11} u_{22}+u_{22}^{2}+u_{22} u_{33}\right) \\
& +8(p-1) u_{13} u_{23} u_{3} u_{123} \\
& -2(p+1) u_{11}^{3} u_{22}-4 u_{11}^{2} u_{22}^{2}+12 u_{11}^{2} u_{23}^{2}+(8 p-20) u_{11} u_{13}^{2} u_{22} \\
& -2(p+1) u_{11} u_{22}^{3}-8(p-1) u_{11} u_{22}^{2} u_{33}+20 u_{11} u_{22} u_{23}^{2} \\
& -2(p-1) u_{11} u_{22} u_{33}^{2}+20(p-1) u_{11} u_{23}^{2} u_{33}-8(p-1) \frac{u_{22}}{u_{11}} u_{13}^{4} \\
& -(8 p-20) u_{13}^{2} u_{22}^{2}+12(p-1) u_{13}^{2} u_{22} u_{33}-8(p-1) u_{13}^{2} u_{23}^{2}
\end{aligned}
$$

with

$$
\begin{aligned}
G(D K)= & \frac{1}{u_{3}^{3}} G_{1}(D K)-G_{2}(D K)-G_{3}(D K) \\
= & 2\left(\frac{u_{3}^{3}}{u_{11}} K_{1}\right)^{2}-2 u_{3}^{3}\left(u_{3} \frac{u_{11}+2 u_{22}}{u_{11}^{1}} u_{111}-4 \frac{u_{13} u_{22}}{u_{11}}-(p-6) u_{13}\right) K_{1} \\
& -2 u_{3}^{3}\left(u_{3} \frac{u_{112}}{u_{11}}-(p-2) u_{23}\right) K_{2} \\
& -2 u_{3}^{3}\left((p-1) u_{3} \frac{u_{113}}{u_{11}}-2(p-1) \frac{u_{11} u_{22}+u_{13}^{2}}{u_{11}}-p u_{33}\right) K_{3} .
\end{aligned}
$$

Now, at the point $x$, by equation (4.3) we have:

$$
u_{33}=-\frac{u_{11}+u_{22}}{p-1} \text {. }
$$

Inserting this into (4.73), we deduce:

$$
\begin{aligned}
|D u|^{2} a^{\alpha \beta} K_{\alpha \beta} & +G(D K) \\
= & -2 \frac{u_{22}}{u_{11}}\left(1+\frac{u_{22}}{u_{11}}\right)\left[u_{3} u_{111}-\frac{2 u_{11}\left(2 u_{11}+u_{22}\right)}{u_{11}+u_{22}} u_{13}\right]^{2} \\
& -2\left(1+\frac{u_{22}}{u_{11}}\right)\left[u_{3} u_{112}-\frac{2 u_{11}^{2}}{u_{11}+u_{22}} u_{23}\right]^{2} \\
& -2(p-1) \frac{u_{22}}{u_{11}}\left[u_{3} u_{113}-2 u_{13}^{2}+\frac{p}{p-1} u_{11}^{2}-\frac{p-2}{p-1} u_{11} u_{22}\right]^{2} \\
& -2(p-1)\left[u_{3} u_{123}-2 u_{13} u_{23}\right]^{2} \\
& -\frac{8 u_{11}}{u_{11}+u_{22}} u_{22}^{2} u_{13}^{2}-\frac{8 u_{22}}{u_{11}+u_{22}} u_{11}^{2} u_{23}^{2}-4(p-2) u_{11}^{2} u_{22}^{2} .
\end{aligned}
$$

Since the level sets of $u$ are strictly convex with respect to the normal $D u$, we have $K>0$. But now $K=F^{33} u_{3} u_{3}|D u|^{-4}=u_{11} u_{22}|D u|^{-2}$; hence $u_{11} u_{22}>0$. So (4.76) yields (4.4) for $2 \leq p<+\infty$. This completes the proof of Theorem 1.3

\section{ACKNOWLEDGMENT}

Part of the work was done while the second author was visiting the Max Planck Institute for Mathematics in the Sciences in January 2008, and he would like to thank this institute for warm hospitality. The second author would also like to thank Professor P. Guan for useful discussions on this subject. The authors would like to thank the referee for his (her) very careful reading and many good suggestions on this paper.

\section{REFERENCES}

[1] L.V. Ahlfors, Conformal invariants: topics in geometric function theory, McGraw-Hill Series in Higher Mathematics. McGraw-Hill Book Co., New York-Desseldorf-Johannesburg, 1973 (pp. 5-6). MR0357743 (50:10211) 
[2] B.J. Bian, P. Guan, X.N. Ma and L. Xu, A microscopic convexity principle for the level sets of solution for nonlinear elliptic partial differential equations, Indiana Univ. Math. J., vol. 60, no. 1, 2011, 101-119.

[3] L. Caffarelli and A. Friedman, Convexity of solutions of some semilinear elliptic equations, Duke Math. J., 52, (1985), 431-455. MR792181 (87a:35028)

[4] L.A. Caffarelli, L. Nirenberg and J. Spruck, Nonlinear second order elliptic equations $I V$ : Starshaped compact Weingarten hypersurfaces, Current topics in partial differential equations, Y.Ohya, K.Kasahara and N.Shimakura (eds.), Kinokunize, Tokyo, 1985, 1-26. MR 1112140

[5] L. Caffarelli and J. Spruck, Convexity properties of solutions to some classical variational problems, Comm. Partial Differ. Equations, 7, (1982), 1337-1379. MR678504 (85f:49062)

[6] P. Cuoghi and P. Salani, Convexity of level sets for solutions to nonlinear elliptic problems in convex rings, Electronic Jour. Diff. Equations, 124, (2006), 1-12. MR2255239 (2007d:35083)

[7] M. P. do Carmo, Differential geometry of curves and surfaces, Translated from the Portuguese. Prentice-Hall, Inc., Englewood Cliffs, N.J., 1976. MR 0394451 (52:15253)

[8] R. Gabriel, An extended principle of the maximum for harmonic functions in 3 dimensions, J. London Math. Soc. 30, (1955), 388-401. MR0072959 (17:358c)

[9] R. Gabriel, A result concerning convex level surfaces of 3-dimensional harmonic functions, J. London Math. Soc. 32, (1957), 286-294. MR0090662 (19:848a)

[10] D. Gilbarg and N. S. Trudinger, Elliptic Partial Differential Equations of Second Order, Springer-Verlag, Berlin, 2nd ed., 1998. MR.1814364 (2001k:35004)

[11] S. Gleason and T. Wolff, Lewy's harmonic gradient maps in higher dimensions. Comm. Partial Diff. Equations, 16, (1991), 1925-1968. MR.1140779 (92k:35062)

[12] A. Greco, Quasi-concavity for semilinear elliptic equations with non-monotone and anisotropic nonlinearities, Boundary Value Problems, 2006, Art. ID 80347, 15 pp. MR2211400 (2006i:35091)

[13] B. Kawohl, Rearrangements and convexity of level sets in PDE, Lectures Notes in Math., 1150, Springer-Verlag, Berlin, 1985. MR810619 (87a:35001)

[14] J.L. Lewis, Capacitary functions in convex rings, Arch. Rational Mech. Anal. 66, (1977), 201-224. MR0477094 (57:16638)

[15] H. Lewy, On the non-vanishing of the Jacobian of a homeomorphism by harmonic gradients, Annals of Math. (2), 88, (1968), 518-529. MR0232007 (38:333)

[16] M. Longinetti, Convexity of the level lines of harmonic functions, (Italian) Boll. Un. Mat. Ital. A 6, (1983), 71-75. MR694746 (84e:31001)

[17] M. Longinetti, On minimal surfaces bounded by two convex curves in parallel planes, J. Diff. Equations, 67, (1987), 344-358. MR884274 (88m:58035)

[18] M. Ortel and M. Schneider, Curvature of level curves of harmonic functions, Canad. Math. Bull. 26, (1983), no. 4, 399-405. MR716578 (84m:31003)

[19] J.P. Rosay and W. Rudin, A maximum principle for sums of subharmonic functions, and the convexity of level sets, Michigan Math. J., 36, (1989), 95-111. MR989939 (90h:31006)

[20] M. Shiffman, On surfaces of stationary area bounded by two circles, or convex curves, in parallel planes, Annals of Math. (2), 63, (1956), 77-90. MR0074695 (17:632d)

[21] G. Talenti, On functions, whose lines of steepest descent bend proportionally to level lines, Ann. Scuola Norm. Sup. Pisa Cl. Sci. 10, (1983), no. 4, 587-605. MR753157 (86b:35021)

[22] N.S. Trudinger, On new isoperimetric inequalities and symmetrization, J. Reine Angew. Math. 488, (1997), 203-220. MR1465371(99a:35076)

Max Planck Institute for Mathematics in the Sciences, Inselstr. 22, D-04103 Leipzig, GERMANY

E-mail address: jjost@mis.mpg.de

Department of Mathematics, University of Science and Technology of China, Hefei, 230026, Anhui Province, People's Republic of China

E-mail address: xinan@ustc.edu.cn

Department of Mathematics, Hezhou University, Hezhou, 542800, Guangxi Province, People's Republic of China

E-mail address: ouqzh@163.com 